\title{
CHARACTERIZING THE COOL KOIs. VIII. PARAMETERS OF THE PLANETS ORBITING KEPLER'S COOLEST DWARFS
}

\author{
Jonathan J. Swift ${ }^{1,5}$, Benjamin T. Montet ${ }^{1,2}$, Andrew Vanderburg ${ }^{2}$, Timothy Morton $^{3}$, Philip S. Muirhead ${ }^{4}$, and \\ JOHN ASHER JOHNSON ${ }^{2}$ \\ ${ }^{1}$ California Institute of Technology, 1200 East California Boulevard, Pasadena, CA 91125, USA \\ ${ }^{2}$ Harvard-Smithsonian Center for Astrophysics, Cambridge, MA 02138, USA \\ ${ }^{3}$ Department of Astrophysical Sciences, Princeton University, 4 Ivy Lane, Peyton Hall, Princeton, NJ 08544, USA \\ ${ }^{4}$ Department of Astronomy, Boston University, 725 Commonwealth Avenue, Boston, MA 02215, USA \\ Received 2014 December 26; accepted 2015 February 26; published 2015 June 22
}

\begin{abstract}
The coolest dwarf stars targeted by the Kepler Mission constitute a relatively small but scientifically valuable subset of the Kepler target stars, and provide a high-fidelity, nearby sample of transiting planetary systems. Using archival Kepler data spanning the entire primary mission, we perform a uniform analysis to extract, confirm, and characterize the transit signals discovered by the Kepler pipeline toward M-type dwarf stars. We recover all but two of the signals reported in a recent listing from the Exoplanet Archive resulting in 163 planet candidates associated with a sample of 104 low-mass stars. We fitted the observed light curves to transit models using a Markov Chain Monte Carlo method and we have made the posterior samples publicly available to facilitate further studies. We fitted empirical transit times to individual transit signals with significantly non-linear ephemerides for accurate recovery of transit parameters and precise measuring of transit timing variations. We also provide the physical parameters for the stellar sample, including new measurements of stellar rotation, allowing the conversion of transit parameters into planet radii and orbital parameters.
\end{abstract}

Key words: methods: statistical - planets and satellites: general - stars: late-type - stars: low-mass

Supporting material: figure sets, FITS file

\section{INTRODUCTION}

NASA's Kepler Space Mission was designed to monitor more than 150,000 stars within a single 115 square degree patch of sky in search of periodic diminutions of light caused by transiting exoplanets (Borucki et al. 2010; Jenkins et al. 2010; Koch et al. 2010). Kepler's great success in discovering transiting exoplanets (Borucki et al. 2011a, 2011b; Batalha et al. 2013; Burke et al. 2014) has revealed that planets are at least as numerous as stars in the Galaxy (Dressing \& Charbonneau 2013; Fressin et al. 2013; Petigura et al. 2013a; Swift et al. 2013; Morton \& Swift 2014). Beyond the sheer number of planets, Kepler has also provided important insights into the characteristics of the transiting planet population. The multi-transit systems reveal highly coplanar multi-planet systems (Lissauer et al. 2011b; Fang \& Margot 2012; Tremaine \& Dong 2012; Ballard \& Johnson 2014; Fabrycky et al. 2014), many of which are in compact configurations (e.g., Lissauer et al. 2011a; Muirhead et al. 2012b; Swift et al. 2013). The period ratios of adjacent transiting planets show an excess just outside of mean motion resonance (Lissauer et al. 2011b; Fabrycky et al. 2014) that may reflect the mechanisms through which these systems formed (Rein 2012; Goldreich \& Schlichting 2014), or else may indicate subsequent evolution of these systems (Lithwick \& Wu 2012; Batygin \& Morbidelli 2013). The typical surface density profile of the protoplanetary disks from which these planets formed can be estimated using the Kepler sample, and implies that either protoplanetary disks contain a large amount of material within $\sim 0.1 \mathrm{AU}$ of the host star (Hansen \& Murray 2012; Chiang \& Laughlin 2013) or that the planets migrated from their birth places further out in the

\footnotetext{
${ }^{5}$ Current address: The Thacher School, 5025 Thacher Road, Ojai, CA 93023, USA.
}

disk (Swift et al. 2013; Schlichting 2014). Another clue regarding the formation mechanisms behind the Kepler planet sample is the radius function - the frequency of planets as a function of their size - which shows unambiguously that there are many more planets with radii smaller than that of Neptune than there are larger ones (Howard et al. 2012; Fressin et al. 2013; Petigura et al. 2013b; Morton \& Swift 2014; ForemanMackey et al. 2014).

Although the vast majority of Kepler target stars are Sun-like $\left(0.8 M_{\odot} \lesssim M_{\star} \lesssim 1.2 M_{\odot}\right), \quad$ several M-type dwarf stars (M dwarfs) have been monitored by Kepler over the course of the primary mission. The initial photometric characterization of the M dwarfs in the Kepler field was known to be inaccurate because the Kepler Input Catalog (KIC) was optimized for Sun-like stars (Brown et al. 2011). However, there have been several efforts to revise the stellar parameters for this sample (e.g., Mann et al. 2012, 2013; Muirhead et al. 2012b, 2014; Dressing \& Charbonneau 2013; Newton et al. 2014). Since the physical parameters of a transiting planet are dependent on the stellar parameters, many exciting results have come from a careful examination of this stellar sample (e.g., Johnson et al. 2011a, 2012; Muirhead et al. 2012a, 2013). The depth of a transit signal is proportional to the square of the relative planet radius, $\delta \propto\left(R_{p} / R_{\star}\right)^{2}$, allowing the detection of smaller planets around these smaller stars. This higher sensitivity to smaller planets allows the planet population around Kepler's M dwarfs to be well-sampled down to $\lesssim 1 R_{\oplus}$, where planets are most prevalent (Morton \& Swift 2014). Furthermore, the theoretical "habitable zone," in which planets have equilibrium temperatures comparable to that of the Earth, is much closer to these cool, faint stars. This increases the transit probability and number of transits per an observing time baseline, thereby allowing the first detection and measurement of the occurrence 
of Earth-sized planets in the habitable zones of stars (Dressing \& Charbonneau 2013; Quintana et al. 2014)

As a supplement to our recent efforts to characterize the lowest mass stars in the Kepler field (Muirhead et al. 2012a, 2014), here we focus on the transit signals in the list of $M$ dwarf Kepler Objects of Interest (KOIs). The following is a uniform treatment of the sample which we use to derive a statistically useful body of information regarding the properties of the planets orbiting Kepler's lowest-mass stars. In Section 2, we introduce the criteria that were used to define our sample, and in Section 3 we follow with a description of the Kepler data products and the preparation of these data for our following analyses. In Section 4, we outline in detail our treatment of the Kepler data including a preliminary characterization of the data with outlier rejection and a Markov Chain Monte Carlo parameter estimation. Also in this section, we search for transit timing variations (TTVs) in the light curve data that may be due to mutual gravitational interactions within multi-planet systems or other effects, and perform custom fits to the transit shapes of those sources with significantly non-linear ephemerides. We present the full ensemble of transit candidates and stellar parameters in Section 6 and conclude in Section 7.

\section{SAMPLE OF PLANET CANDIDATES}

Our list of cool planet host stars is drawn from a recent KOI list available through the Exoplanet Archive (Akeson et al. 2013, downloaded on 2014 September 18) which included the planet candidate sample derived from quarters 1 through 12 of the Kepler Mission (Rowe et al. 2015). A total of 4228 planet transit signals toward 3250 targets were selected from the KOI list with dispositions of either "candidate" or "confirmed," comprising a high-fidelity catalog of exoplanets (see, e.g., Morton \& Johnson 2011; Morton 2012; Fressin et al. 2013). From this list of candidates, we choose those with host star color $K_{p}-J>2$ and $K_{p}>14$ as a cut for M dwarfs (Mann et al. 2012). We also include six stars with $r-J>2.0$ from the study by Muirhead et al. (2014) which pass our red criterion but not our faint criterion: KOI-314, KOI-641, KOI-1725, KOI-3444, KOI-3497, and KOI-4252. Finally, we also include the new planet discovered by Muirhead et al. (2015), KOI-2704.03, or Kepler-445d.

We cross-match this full list with the list presented by Muirhead et al. (2014) in which near-infrared spectra for 106 stars toward 103 KOIs are presented. Two of the sources in that list are now categorized as false positives: KOI-1459 and KOI3090. Another binary system, KOI-4463, consists of stars that appear earlier than M0 in Muirhead et al. (2014), and the KOI is not included in the Dressing \& Charbonneau (2013) catalog. We leave these three targets off our list. We also exclude from further consideration a known $\mathrm{M}$ dwarf/white dwarf binary in the list (KOI-256 Muirhead et al. 2013), and the giant star KOI-977 (Muirhead et al. 2014). Lastly, we leave of KOI1686.01 and KOI-1408.02 due to inadequate signal retrieval (see Section 3.2). We therefore consider $97 \mathrm{cool} \mathrm{KOIs} \mathrm{from} \mathrm{the}$ Muirhead et al. (2014) list incorporating all 64 targets in the KOI catalog of Dressing \& Charbonneau (2013), save one other now-known false positive, KOI-1164.

The newest release of KOIs (Mullally et al. 2015) postdates both the Muirhead et al. (2014) and Dressing \& Charbonneau (2013) catalogs, and so we also cross matched our KOI list against the full catalog of Dressing \& Charbonneau (2013) to find seven additional cool stars with candidate transit signals:
KOI-2480, KOI-2793 KOI-3102, KOI-3094, KOI-5228, KOI5359, and KOI-5692. These targets are some of the smallest and longest-period planet candidates in our list and offer exciting possibilities for follow-up observations. We note that our target list is not comprehensive as there are many other independent searches for planet signals in the Kepler data (e.g., Fischer et al. 2012; Ofir \& Dreizler 2013; Sanchis 2014) and efforts are ongoing.

The final sample we consider for further characterization consists of 163 planet signals toward 104 cool stars observed by Kepler. A majority of the stars in this sample (74) show single transit signals, while we find 12 double systems, 10 triple systems, 5 quadruple systems, and 3 quintuple systems. However, the majority of planet candidates, $54.6 \%$, are in multi-transiting systems. The multi-planet systems have a higher probability of being true planetary systems due to a paucity of astrophysical false positive scenarios that could produce multiple, independent transit-like signals within a single Kepler aperture (e.g., Lissauer et al. 2014; Rowe et al. 2014) while also passing the data validation pipeline ( $\mathrm{Wu}$ et al. 2010).

\section{DATA PREPARATION}

\subsection{Kepler Data}

The targets in our sample were observed over the entire course of the Kepler mission. However, in Quarter 0 only three cool KOI targets were observed. Over the rest of the mission, an average of $87 \%$ of the targets in our sample were observed each quarter producing an average of 53,366 long cadence data per target and a total of 5.6 million long cadence photometric measurements for our sample. None of the targets in our sample were observed in short cadence mode until quarter 6 when $9.6 \%$ of the targets made the short cadence target list. This fraction rose fairly steadily for the rest of the mission up to quarter 17 when $24 \%$ of the cool KOIs were observed in short cadence mode producing a total of 25 million short cadence data.

We obtained the light curve data through the the Barbara A. Mikulski Archive for Space Telescopes ${ }^{6}$ (MAST) using Data Release 21 for Quarters 0 through 14, release 20 for Quarter 15, and releases 22 and 23 for Quarters 16 and 17, respectively. For all Kepler data header keyword definitions, we refer the reader to the Kepler Archive Manual. ${ }^{7}$ We consider only those data with SAP QUALITY values equal to 0 . This excludes data that were taken under non-optimal circumstances or were flagged for other reasons. On average, this resulted in a rejection of about $12.5 \%$ of long cadence data per target and $6.2 \%$ of short cadence data per target.

For each KOI, both the Pre-search Data Conditioning (PDCSAP; Smith et al. 2012; Stumpe et al. 2012) and Simple Aperture Photometry (SAP) data were examined. The SAP data were cotrended using the first five cotrending basis vectors available through the MAST website, and then deblended using the FLFRCSAP and CROWDSAP header keywords. In all cases, our calibrated SAP data appeared very similar or nearly identical to the PDSCAP data and as our default we use the PDCSAP data for all KOIs for the sake of uniformity.

Before addressing the transit signals, we first look at the raw data for anomalies, trends, and other potential problems.

\footnotetext{
https://archive.stsci.edu/kepler

7 See http://archive.stsci.edu/kepler/manuals/archive_manual.pdf.
} 
$\mathrm{KOI} 247=\mathrm{KIC} 11852982$
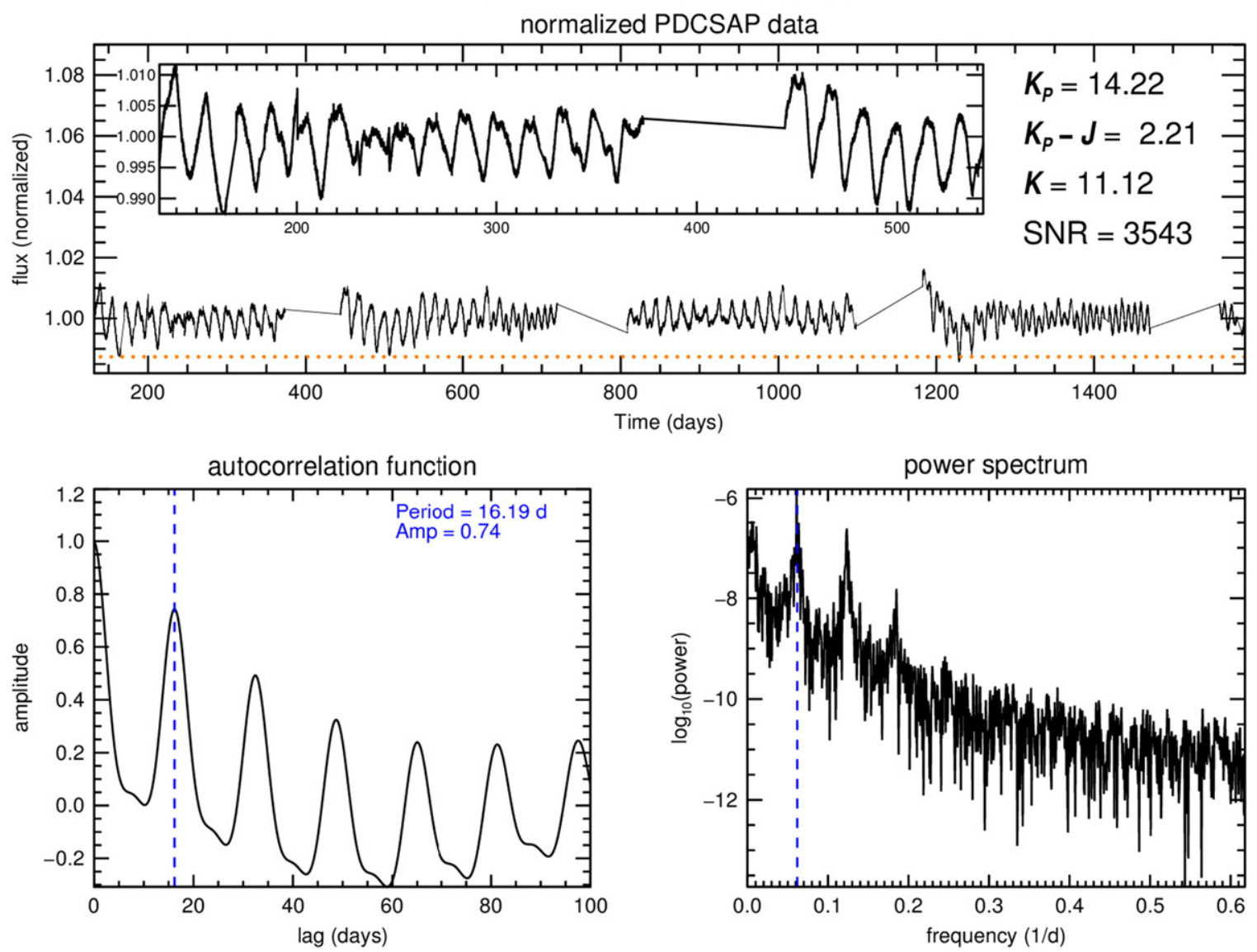

Figure 1. Example of a diagnostic plot for the long cadence data of KOI-247 showing the out of transit data characteristics including the signal to noise of the light curve and absolute photometry. The top panel shows the entire span of the long cadence data set with a zoom in window of the first 400 days. The transit times are marked on the upper panel plot color coded by KOI planet candidate number assignment $(.01=$ orange; $.02=$ purple; $.03=$ gray; $.04=$ cyan; $.05=$ magenta). The lower panels show periodicities in the out of transit data via the auto-correlation function (lower left) and Fourier transform (lower right) from which we estimate the stellar rotation period. The vertical lines (dashed blue) denote the peak of the auto-correlation function and its corresponding frequency.

(The complete figure set (104 images) is available.)

Figure 1 shows an example of one of our diagnostic plots that displays the entire time series of data, a zoom in of a small portion of the data, and photometry information. A normalized flux series is created for each KOI in our list by concatenating all of the available data normalized by the median flux value of each quarter. We then subtract the median flux of the combined series and blank out any transit signals using the durations and ephemerides provided by the Exoplanet Archive. These data are then gridded onto a uniform time series and zeroed at values where data were missing. Periods were searched out to 100 days using both an auto-correlation and a Fourier transform. The normalized light curves, auto-correlation functions, and spectral power density were then inspected by eye. In a majority of cases where periodic signatures were seen, they are interpreted as modulations due to the combination of stellar rotation and a non-uniform stellar surface brightness.

\subsection{Extracting Transit Signals}

Each of the 163 planet signals described above was extracted from the full Kepler light curve by fitting a linear drift to the out of transit data extending two transit durations before the beginning of ingress and two durations after egress. For KOIs with multiple candidate planet signals, all other signals were blanked from the time series data before extraction. The transit times and durations used in this process were taken from the Exoplanet Archive. Some sources with large TTVs (such as KOI-314) required a larger buffer. Linear ephemerides were assumed for each of the transit signals in the extraction process. However, a small buffer of $10 \%$ of the reported transit duration was used to account for any potential TTVs or errors in the values reported by the Exoplanet Archive. The rms value of the residuals to the linear trend is recorded and applied to all of the data from each transit event as the relative flux error.

Next, each transit signal was confirmed using a box-leastsquared algorithm (BLS; Kovács et al. 2002) optimized to oversample the projected BLS peak width by a factor of three (see Ofir 2014). This typically produced convincing transit signals with durations and ephemerides that were generally in agreement with the values of the Exoplanet Archive. However, there were a few exceptions. KOI-1686.01 and KOI-1408.02 ${ }^{8}$ do not show a convincing transit signal and have been left off our list. Also, the period reported for KOI-1725 was found to

\footnotetext{
8 In the latest release from the Exoplanet Archive, these sources are designated as False Positives (Mullally et al. 2015).
} 

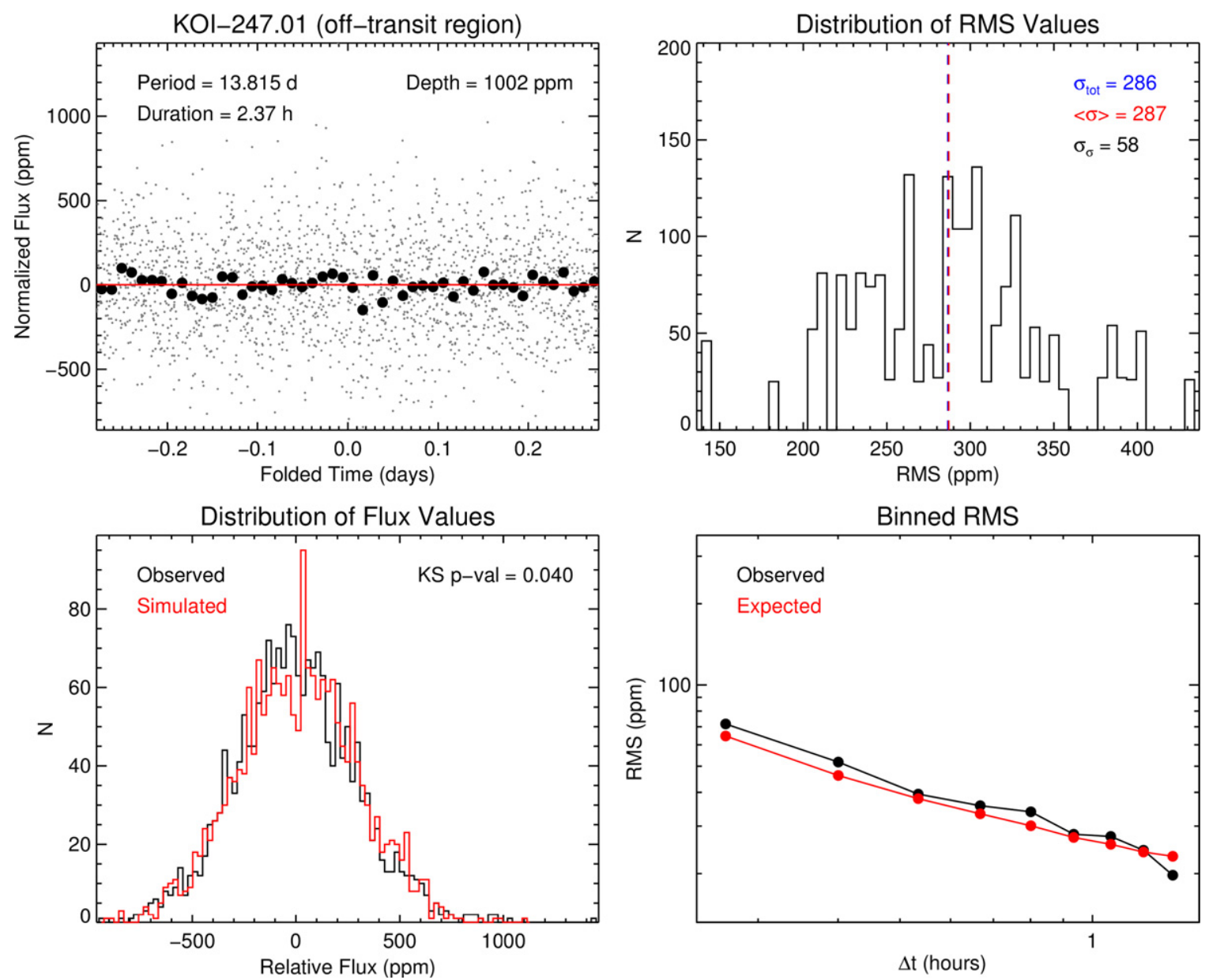

Figure 2. (Top left) The adjacent, transit-free section of the light curve for the specified KOI is shown folded on the period of the planet transit signal. The calibrated Kepler data are shown as small dots, and binned data are plotted as larger dots to reveal more subtle structure. (Top right) The distribution of rms values derived from the detrending process are shown in histrogram form. The rms of the folded data, $\sigma_{\text {tot }}$, is depicted with the blue dotted line; the mean of the rms values derived from the detrending process, $\langle\sigma\rangle$, is shown as the dotted red line; and the spread in the detrend derived rms values, $\sigma_{\sigma}$, is also displayed. (Bottom left) Histogram of the data from the top left panel is shown and compared with a histogram of values drawn from a normal distribution with zero mean and a standard deviation equal to the rms of the data. The results from a two-sided Kolmogorov-Smirnov test show the probability that the two distributions were drawn from the same parent sample. (Bottom right) The phase folded data are binned on a series of timescales, $\Delta t$, starting with the smallest bin which will include at least 20 points and stepping up in 10 bins to one half of the transit duration as reported by the Kepler team. This curve is shown in relation to the expected trend (e.g., Winn et al. 2008).

(The complete figure set (163 images) is available.)

be approximately nine minutes off, necessitating an independent period search to adequately retrieve this signal. In cases where a transit signal was apparent in long cadence data, but problematic or not clearly seen in the short cadence data (typically due to a paucity of short cadence data), the transit parameters derived from the long cadence data were applied to the short cadence data. Examples of extracted transit signals are shown in Section 4.

Correlated noise produced by either instrumental or astrophysical phenomena can have a significant affect on the interpretation of astronomical light curve data (see, e.g., Pont et al. 2006; Carter \& Winn 2009). Therefore, in addition to the transit extraction, a section of the light curve with no transit signal was extracted in exactly the same manner as the transit signal, but according to a mid-transit time advanced by five times the reported transit duration. This produced a transit-free section of the light curve immediately adjacent to the extracted transit events. Figure 2 shows one example of a "blank" extraction as well as the basic analyses we use to assess the noise properties of our data (see caption for more details). We find that the distribution of data values for each KOI can be reasonably described by a single parameter, $\sigma$, and compares well with synthetic, Gaussian distributed data (typical KS $p$ values $\gtrsim 0.01)$. The fact that the noise properties of our data sample appear to be nearly Gaussian can be attributed to a variety of factors. One dominant effect is that the stars in our sample are by design faint, meaning that the photon noise is higher than for the rest of the sample, which can mask subtler, correlated phenomena. Also, the astrophysical noise from M dwarf light curves is typically caused by inhomogeneities in the stellar surface brightness coupled with stellar rotation rather than pulsation modes (see, e.g., Rodríguez-López et al. 2012). The stellar rotation timescales are typically much longer than the transit durations, and so these effects are adequately corrected with our detrending process. We therefore do not consider the effects of correlated noise in later analyses.

The final step in the preparation of our light curves is outlier rejection. This procedure removes astrophysical (e.g., flares) and instrumental effects not accounted for in the above procedures as well as points that were not adequately 
Table 1

Transit Parameters for Long Cadence Fits

\begin{tabular}{|c|c|c|c|c|c|c|c|c|c|c|}
\hline KOI & $\begin{array}{c}P \\
\text { (days) }\end{array}$ & $\begin{array}{l}\delta P \\
(\mathrm{~s})\end{array}$ & $\begin{array}{c}t_{0} \\
\text { BJD-2454833 }\end{array}$ & $\begin{array}{l}\delta t_{0} \\
(\mathrm{~s})\end{array}$ & $\begin{array}{c}R_{p} / R_{\star} \\
(\%)\end{array}$ & $\begin{array}{c}\delta R_{p} / R_{\star} \\
(\%) \\
\end{array}$ & $\begin{array}{l}\tau_{\text {tot }} \\
(\mathrm{hr})\end{array}$ & $\begin{array}{c}\delta \tau_{\text {tot }} \\
\text { (minutes) }\end{array}$ & $b$ & $\delta b$ \\
\hline 247.01 & 13.815050 & 1.67 & 858.062061 & 50.82 & 2.909 & 0.225 & 2.2848 & 5.400 & 0.45 & 0.31 \\
\hline $248.01^{\mathrm{a}}$ & 7.203854 & 0.51 & 861.856433 & 30.55 & 4.105 & 0.111 & 2.5224 & 2.808 & 0.36 & 0.24 \\
\hline $248.02^{\mathrm{a}}$ & 10.912760 & 1.56 & 868.268258 & 57.09 & 3.338 & 0.234 & 2.5104 & 5.544 & 0.38 & 0.28 \\
\hline 248.03 & 2.576571 & 0.22 & 860.071548 & 36.26 & 2.677 & 0.217 & 1.7088 & 4.176 & 0.47 & 0.32 \\
\hline 248.04 & 18.596124 & 4.60 & 866.513352 & 90.03 & 2.744 & 0.231 & 2.3376 & 6.768 & 0.47 & 0.32 \\
\hline 249.01 & 9.549275 & 0.43 & 863.305359 & 17.86 & 4.046 & 0.225 & 1.5960 & 2.952 & 0.45 & 0.30 \\
\hline $250.01^{\mathrm{a}}$ & 12.283005 & 0.80 & 845.963352 & 27.46 & 5.067 & 0.213 & 2.7624 & 3.888 & 0.41 & 0.30 \\
\hline $250.02^{\mathrm{a}}$ & 17.251179 & 1.74 & 857.185157 & 43.44 & 4.367 & 0.266 & 1.9608 & 5.184 & 0.51 & 0.29 \\
\hline 250.03 & 3.543902 & 0.79 & 859.215985 & 95.76 & 1.838 & 0.154 & 2.1216 & 7.272 & 0.43 & 0.30 \\
\hline 250.04 & 46.827733 & 8.12 & 885.988374 & 75.71 & 3.907 & 0.227 & 1.8696 & 5.688 & 0.46 & 0.30 \\
\hline 251.01 & 4.164384 & 0.15 & 858.211584 & 14.39 & 4.678 & 0.194 & 1.8192 & 2.664 & 0.42 & 0.27 \\
\hline 251.02 & 5.774417 & 2.35 & 860.544378 & 153.87 & 1.549 & 0.109 & 1.8288 & 8.064 & 0.48 & 0.33 \\
\hline 252.01 & 17.604618 & 2.09 & 857.078072 & 49.29 & 4.641 & 0.340 & 3.6984 & 8.208 & 0.58 & 0.27 \\
\hline 253.01 & 6.383165 & 0.53 & 859.985599 & 33.82 & 4.271 & 0.287 & 1.8120 & 4.032 & 0.43 & 0.29 \\
\hline 253.02 & 20.618078 & 12.75 & 867.149460 & 318.82 & 2.382 & 0.212 & 3.2160 & 15.912 & 0.48 & 0.33 \\
\hline 254.01 & 2.455241 & 0.01 & 863.199607 & 1.40 & 19.063 & 0.818 & 1.8336 & 1.152 & 0.56 & 0.04 \\
\hline 255.01 & 27.522008 & 2.78 & 850.351246 & 43.24 & 4.583 & 0.199 & 4.1208 & 4.680 & 0.37 & 0.25 \\
\hline 255.02 & 13.602938 & 12.77 & 861.366322 & 369.59 & 1.375 & 0.127 & 2.8056 & 15.264 & 0.48 & 0.33 \\
\hline $314.01^{\mathrm{a}}$ & 13.781096 & 0.77 & 853.126068 & 22.01 & 2.514 & 0.117 & 2.3016 & 3.024 & 0.49 & 0.31 \\
\hline $314.02^{\mathrm{a}}$ & 23.088964 & 2.42 & 863.679273 & 44.00 & 2.277 & 0.113 & 1.7736 & 3.456 & 0.46 & 0.30 \\
\hline $314.03^{\mathrm{a}}$ & 10.313231 & 4.37 & 855.441694 & 149.86 & 1.084 & 0.066 & 1.9992 & 6.336 & 0.48 & 0.33 \\
\hline $463.01^{\mathrm{a}}$ & 18.477644 & 1.32 & 868.940991 & 29.86 & 4.923 & 0.160 & 1.8288 & 2.808 & 0.35 & 0.24 \\
\hline 478.01 & 11.023478 & 0.68 & 854.569829 & 22.75 & 4.033 & 0.192 & 1.3968 & 3.024 & 0.40 & 0.29 \\
\hline 531.01 & 3.687470 & 0.03 & 860.437248 & 3.85 & 5.677 & 0.601 & 1.0272 & 1.512 & 0.31 & 0.20 \\
\hline 571.01 & 7.267302 & 0.89 & 857.440913 & 49.58 & 2.506 & 0.133 & 2.2872 & 4.680 & 0.43 & 0.32 \\
\hline 571.02 & 13.343016 & 2.20 & 857.396606 & 63.93 & 2.737 & 0.171 & 2.7600 & 5.328 & 0.47 & 0.32 \\
\hline 571.03 & 3.886785 & 0.46 & 860.160360 & 49.69 & 2.125 & 0.147 & 1.9008 & 4.824 & 0.47 & 0.32 \\
\hline 571.04 & 22.407609 & 6.06 & 870.846301 & 110.16 & 2.468 & 0.135 & 3.3288 & 7.920 & 0.47 & 0.31 \\
\hline 571.05 & 129.943525 & 147.13 & 826.551452 & 441.38 & 2.111 & 0.168 & 5.8080 & 23.400 & 0.49 & 0.34 \\
\hline 596.01 & 1.682696 & 0.10 & 860.358616 & 24.01 & 2.501 & 0.123 & 1.4184 & 2.664 & 0.41 & 0.28 \\
\hline 641.01 & 14.851847 & 1.58 & 861.184378 & 47.21 & 3.263 & 0.153 & 3.3552 & 4.752 & 0.39 & 0.27 \\
\hline 739.01 & 1.287077 & 0.10 & 862.263195 & 31.16 & 2.679 & 0.107 & 1.4568 & 3.024 & 0.44 & 0.32 \\
\hline 781.01 & 11.598224 & 1.21 & 853.092509 & 43.37 & 5.152 & 0.319 & 2.5224 & 4.968 & 0.43 & 0.28 \\
\hline 812.01 & 3.340220 & 0.28 & 860.063988 & 34.38 & 4.009 & 0.169 & 1.9224 & 3.312 & 0.41 & 0.28 \\
\hline 812.02 & 20.060375 & 5.21 & 869.639153 & 107.38 & 3.806 & 0.220 & 3.3552 & 7.488 & 0.41 & 0.28 \\
\hline 812.03 & 46.184176 & 22.54 & 904.183430 & 193.55 & 3.744 & 0.207 & 4.7712 & 12.312 & 0.45 & 0.32 \\
\hline 812.04 & 7.825033 & 3.73 & 856.457413 & 194.71 & 2.321 & 0.198 & 2.2248 & 11.232 & 0.47 & 0.33 \\
\hline 817.01 & 23.967927 & 6.72 & 857.325190 & 131.08 & 3.411 & 0.183 & 3.8664 & 9.720 & 0.49 & 0.31 \\
\hline 817.02 & 8.295585 & 1.47 & 840.919728 & 74.93 & 2.868 & 0.252 & 1.1616 & 6.120 & 0.46 & 0.32 \\
\hline 818.01 & 8.114381 & 0.89 & 857.947143 & 47.90 & 3.899 & 0.212 & 2.2608 & 4.608 & 0.45 & 0.29 \\
\hline 854.01 & 56.056171 & 21.46 & 817.783856 & 162.12 & 3.946 & 0.221 & 4.5552 & 12.456 & 0.50 & 0.31 \\
\hline $886.01^{\mathrm{a}}$ & 8.010828 & 2.07 & 859.135626 & 116.70 & 3.586 & 0.318 & 2.5080 & 9.216 & 0.56 & 0.30 \\
\hline $886.02^{\mathrm{a}}$ & 12.071357 & 6.10 & 867.740134 & 237.23 & 2.433 & 0.173 & 4.5336 & 10.584 & 0.31 & 0.23 \\
\hline 886.03 & 20.995946 & 10.18 & 845.184612 & 215.08 & 2.553 & 0.183 & 2.9544 & 12.168 & 0.48 & 0.33 \\
\hline $898.01^{\mathrm{a}}$ & 9.770453 & 1.29 & 849.875896 & 55.18 & 4.370 & 0.165 & 2.4216 & 4.248 & 0.41 & 0.28 \\
\hline 898.02 & 5.169805 & 0.85 & 865.384993 & 67.98 & 3.207 & 0.218 & 2.2200 & 6.912 & 0.51 & 0.32 \\
\hline 898.03 & 20.090234 & 5.88 & 871.224471 & 120.16 & 3.701 & 0.263 & 3.6840 & 10.512 & 0.50 & 0.32 \\
\hline 899.01 & 7.113716 & 0.99 & 864.355699 & 58.81 & 2.701 & 0.164 & 2.1624 & 5.184 & 0.47 & 0.30 \\
\hline 899.02 & 3.306546 & 0.52 & 861.814312 & 60.46 & 2.163 & 0.163 & 1.8168 & 5.832 & 0.46 & 0.32 \\
\hline 899.03 & 15.368446 & 3.41 & 854.339483 & 95.93 & 2.652 & 0.173 & 2.4936 & 7.200 & 0.47 & 0.32 \\
\hline 936.01 & 9.467811 & 0.52 & 869.566887 & 23.44 & 4.459 & 0.146 & 2.4552 & 2.808 & 0.35 & 0.24 \\
\hline 936.02 & 0.893042 & 0.04 & 861.475877 & 17.66 & 2.645 & 0.132 & 1.0968 & 2.376 & 0.43 & 0.29 \\
\hline 947.01 & 28.599142 & 4.45 & 847.706511 & 66.99 & 3.859 & 0.141 & 3.6744 & 5.400 & 0.38 & 0.27 \\
\hline 952.01 & 5.901277 & 0.65 & 861.858407 & 48.55 & 3.943 & 0.153 & 2.2272 & 4.320 & 0.43 & 0.29 \\
\hline $952.02^{\mathrm{a}}$ & 8.752103 & 1.67 & 862.049430 & 82.39 & 3.819 & 0.316 & 2.3328 & 8.208 & 0.55 & 0.30 \\
\hline 952.03 & 22.780766 & 4.08 & 861.404117 & 77.15 & 4.455 & 0.118 & 3.1992 & 4.824 & 0.38 & 0.27 \\
\hline 952.04 & 2.896015 & 0.94 & 860.512106 & 153.00 & 1.957 & 0.194 & 2.0496 & 10.944 & 0.43 & 0.30 \\
\hline 952.05 & 0.742962 & 0.23 & 860.636153 & 122.51 & 1.400 & 0.122 & 1.2984 & 6.768 & 0.46 & 0.32 \\
\hline 961.01 & 1.213770 & 0.03 & 862.333219 & 9.88 & 4.211 & 0.268 & 0.5448 & 1.512 & 0.45 & 0.30 \\
\hline 961.02 & 0.453288 & 0.00 & 861.396478 & 3.98 & 3.854 & 0.130 & 0.5040 & 0.792 & 0.35 & 0.24 \\
\hline 961.03 & 1.865114 & 0.08 & 861.186344 & 18.22 & 3.615 & 0.274 & 0.4512 & 2.088 & 0.44 & 0.31 \\
\hline 1078.01 & 3.353728 & 0.35 & 862.627731 & 44.73 & 3.536 & 0.256 & 1.5672 & 5.040 & 0.47 & 0.33 \\
\hline 1078.02 & 6.877453 & 0.76 & 861.094063 & 48.02 & 3.977 & 0.220 & 1.3248 & 4.104 & 0.44 & 0.31 \\
\hline
\end{tabular}


Table 1

(Continued)

\begin{tabular}{|c|c|c|c|c|c|c|c|c|c|c|}
\hline KOI & $\begin{array}{c}P \\
\text { (days) }\end{array}$ & $\begin{array}{l}\delta P \\
(\mathrm{~s})\end{array}$ & $\begin{array}{c}t_{0} \\
\text { BJD-2454833 }\end{array}$ & $\begin{array}{l}\delta t_{0} \\
(\mathrm{~s})\end{array}$ & $\begin{array}{c}R_{p} / R_{\star} \\
(\%)\end{array}$ & $\begin{array}{c}\delta R_{p} / R_{\star} \\
(\%)\end{array}$ & $\begin{array}{l}\tau_{\text {tot }} \\
(\mathrm{hr})\end{array}$ & $\begin{array}{c}\delta \tau_{\text {tot }} \\
\text { (minutes) }\end{array}$ & $b$ & $\delta b$ \\
\hline 1078.03 & 28.464536 & 7.56 & 869.653492 & 114.85 & 4.035 & 0.200 & 2.8272 & 7.848 & 0.43 & 0.30 \\
\hline 1085.01 & 7.717951 & 3.44 & 864.639733 & 218.11 & 1.679 & 0.153 & 2.2776 & 11.880 & 0.46 & 0.32 \\
\hline 1141.01 & 5.728131 & 1.70 & 862.652205 & 115.59 & 2.534 & 0.156 & 2.0208 & 7.128 & 0.47 & 0.32 \\
\hline 1146.01 & 7.097120 & 2.63 & 860.244823 & 161.55 & 1.897 & 0.136 & 2.2536 & 9.792 & 0.47 & 0.33 \\
\hline 1201.01 & 2.757592 & 0.43 & 861.053399 & 80.52 & 2.227 & 0.139 & 1.3032 & 4.248 & 0.46 & 0.32 \\
\hline 1393.01 & 1.694740 & 0.11 & 972.242566 & 24.30 & 3.679 & 0.170 & 1.6800 & 3.312 & 0.45 & 0.30 \\
\hline 1397.01 & 6.247032 & 0.74 & 969.118946 & 42.16 & 3.878 & 0.182 & 1.4376 & 3.816 & 0.44 & 0.31 \\
\hline 1408.01 & 14.534055 & 4.46 & 857.599004 & 121.33 & 2.163 & 0.132 & 3.3336 & 7.632 & 0.46 & 0.30 \\
\hline 1422.01 & 5.841635 & 0.84 & 866.127692 & 59.12 & 3.587 & 0.261 & 1.9464 & 5.328 & 0.44 & 0.30 \\
\hline 1422.02 & 19.850252 & 5.28 & 848.261297 & 112.57 & 3.837 & 0.312 & 2.8968 & 8.424 & 0.45 & 0.30 \\
\hline 1422.03 & 10.864435 & 4.70 & 869.906646 & 185.64 & 2.624 & 0.318 & 2.1936 & 14.400 & 0.48 & 0.34 \\
\hline 1422.04 & 63.336340 & 53.61 & 795.964581 & 297.62 & 3.164 & 0.252 & 3.4944 & 15.408 & 0.46 & 0.32 \\
\hline 1422.05 & 34.141951 & 24.69 & 853.022724 & 340.15 & 2.658 & 0.306 & 3.2760 & 21.528 & 0.47 & 0.33 \\
\hline 1427.01 & 2.613018 & 0.50 & 862.145224 & 76.69 & 2.405 & 0.150 & 1.8456 & 5.760 & 0.47 & 0.32 \\
\hline 1649.01 & 4.043551 & 1.15 & 859.463541 & 106.02 & 1.831 & 0.177 & 1.4856 & 7.632 & 0.44 & 0.32 \\
\hline 1681.01 & 6.939112 & 2.21 & 860.005991 & 121.03 & 2.397 & 0.226 & 2.2488 & 10.296 & 0.45 & 0.31 \\
\hline 1681.02 & 1.992809 & 0.66 & 861.073462 & 146.79 & 1.568 & 0.162 & 1.6440 & 9.864 & 0.44 & 0.31 \\
\hline 1681.03 & 3.531068 & 1.32 & 861.721450 & 160.98 & 1.706 & 0.192 & 1.5096 & 10.368 & 0.46 & 0.33 \\
\hline 1702.01 & 1.538181 & 0.13 & 880.916015 & 33.51 & 2.766 & 0.237 & 1.0800 & 4.104 & 0.43 & 0.30 \\
\hline 1725.01 & 9.878652 & 0.89 & 859.552053 & 43.38 & 3.711 & 0.182 & 1.9248 & 3.096 & 0.42 & 0.29 \\
\hline 1843.01 & 4.194497 & 0.40 & 847.198436 & 43.57 & 2.515 & 0.202 & 1.7760 & 5.904 & 0.52 & 0.33 \\
\hline 1843.02 & 6.355838 & 2.74 & 843.001063 & 189.46 & 1.220 & 0.123 & 1.4904 & 9.576 & 0.48 & 0.33 \\
\hline 1867.01 & 2.549564 & 0.30 & 861.781789 & 48.16 & 2.216 & 0.101 & 1.6512 & 3.816 & 0.45 & 0.32 \\
\hline 1867.02 & 13.969499 & 1.68 & 844.614392 & 50.81 & 3.178 & 0.194 & 1.3584 & 4.392 & 0.45 & 0.30 \\
\hline 1867.03 & 5.212318 & 1.20 & 857.220810 & 86.34 & 2.007 & 0.154 & 2.1672 & 6.192 & 0.48 & 0.32 \\
\hline 1868.01 & 17.760788 & 2.29 & 865.699188 & 52.22 & 3.525 & 0.197 & 1.6248 & 4.536 & 0.45 & 0.32 \\
\hline 1879.01 & 22.085589 & 3.93 & 974.483549 & 56.76 & 5.267 & 0.371 & 2.2080 & 5.976 & 0.47 & 0.30 \\
\hline 1880.01 & 1.151167 & 0.05 & 861.920492 & 16.61 & 2.355 & 0.083 & 1.0488 & 1.944 & 0.50 & 0.33 \\
\hline 1902.01 & 137.864485 & 24.66 & 862.133272 & 74.31 & 4.050 & 0.719 & 1.7112 & 9.432 & 0.44 & 0.34 \\
\hline 1907.01 & 11.350118 & 1.81 & 860.450521 & 69.52 & 3.333 & 0.206 & 2.2992 & 5.976 & 0.46 & 0.31 \\
\hline 2006.01 & 3.273459 & 0.43 & 861.155294 & 54.02 & 1.543 & 0.070 & 1.6920 & 3.672 & 0.49 & 0.32 \\
\hline 2036.01 & 8.410996 & 2.19 & 857.144315 & 106.06 & 2.616 & 0.236 & 2.3592 & 8.136 & 0.43 & 0.30 \\
\hline 2036.02 & 5.795327 & 3.26 & 855.404252 & 196.06 & 1.787 & 0.178 & 2.3040 & 11.664 & 0.47 & 0.33 \\
\hline 2057.01 & 5.945659 & 1.28 & 865.310547 & 88.88 & 1.952 & 0.184 & 2.2392 & 7.344 & 0.41 & 0.29 \\
\hline 2058.01 & 1.523729 & 0.23 & 861.485510 & 60.77 & 1.769 & 0.165 & 1.3776 & 6.480 & 0.43 & 0.30 \\
\hline 2090.01 & 5.132484 & 0.79 & 973.887826 & 52.79 & 2.847 & 0.161 & 1.4952 & 4.464 & 0.45 & 0.30 \\
\hline 2130.01 & 16.855930 & 5.54 & 863.073317 & 168.40 & 3.012 & 0.229 & 2.4792 & 13.608 & 0.50 & 0.34 \\
\hline 2156.01 & 2.852353 & 0.25 & 859.393812 & 36.64 & 3.640 & 0.281 & 0.7728 & 3.384 & 0.44 & 0.31 \\
\hline 2179.01 & 14.871553 & 4.24 & 970.498340 & 105.09 & 2.969 & 0.170 & 2.4864 & 6.840 & 0.46 & 0.31 \\
\hline 2179.02 & 2.732765 & 0.43 & 971.700991 & 54.36 & 2.445 & 0.151 & 1.0488 & 3.816 & 0.46 & 0.32 \\
\hline 2191.01 & 8.847876 & 2.52 & 847.979545 & 125.58 & 1.987 & 0.184 & 2.2728 & 9.648 & 0.38 & 0.28 \\
\hline 2238.01 & 1.646802 & 0.22 & 861.594051 & 54.26 & 1.603 & 0.137 & 1.2600 & 5.472 & 0.42 & 0.30 \\
\hline 2306.01 & 0.512407 & 0.04 & 860.882688 & 32.66 & 1.729 & 0.119 & 1.0944 & 2.952 & 0.45 & 0.30 \\
\hline 2329.01 & 1.615360 & 0.21 & 861.312567 & 53.06 & 2.159 & 0.237 & 1.0896 & 6.120 & 0.43 & 0.32 \\
\hline 2347.01 & 0.588001 & 0.05 & 861.179765 & 33.63 & 1.657 & 0.105 & 1.1352 & 3.312 & 0.46 & 0.32 \\
\hline 2417.01 & 47.705251 & 42.29 & 1052.717647 & 307.98 & 3.393 & 0.352 & 5.6904 & 21.744 & 0.50 & 0.33 \\
\hline 2418.01 & 86.829090 & 113.66 & 883.909263 & 507.11 & 2.674 & 0.281 & 6.1656 & 32.760 & 0.49 & 0.34 \\
\hline 2453.01 & 1.530516 & 0.15 & 860.392109 & 40.99 & 2.564 & 0.244 & 0.5664 & 3.672 & 0.48 & 0.34 \\
\hline 2480.01 & 0.666826 & 0.06 & 861.216259 & 39.08 & 2.247 & 0.186 & 0.7680 & 3.744 & 0.46 & 0.32 \\
\hline 2542.01 & 0.727330 & 0.11 & 844.428343 & 69.24 & 1.810 & 0.139 & 0.9360 & 4.608 & 0.46 & 0.32 \\
\hline 2626.01 & 38.097253 & 23.14 & 863.851953 & 268.07 & 2.965 & 0.263 & 3.3552 & 16.560 & 0.46 & 0.32 \\
\hline 2650.01 & 34.989404 & 29.93 & 879.002425 & 358.94 & 2.306 & 0.186 & 4.4328 & 16.848 & 0.45 & 0.32 \\
\hline 2650.02 & 7.054276 & 3.27 & 862.780386 & 217.51 & 1.848 & 0.196 & 1.7640 & 11.592 & 0.46 & 0.33 \\
\hline 2662.01 & 2.104337 & 0.36 & 861.414256 & 71.87 & 1.504 & 0.103 & 1.0320 & 4.536 & 0.47 & 0.33 \\
\hline 2704.01 & 4.871225 & 0.68 & 1068.916908 & 42.36 & 10.241 & 0.521 & 1.5552 & 4.464 & 0.33 & 0.24 \\
\hline 2704.02 & 2.984151 & 0.69 & 1064.175129 & 64.21 & 6.679 & 0.352 & 1.3272 & 4.536 & 0.40 & 0.28 \\
\hline 2704.03 & 8.152687 & 9.42 & 1063.603281 & 305.72 & 5.227 & 0.823 & 2.3928 & 28.800 & 0.48 & 0.36 \\
\hline 2705.01 & 2.886761 & 0.26 & 1064.090471 & 29.89 & 2.350 & 0.233 & 0.9024 & 3.672 & 0.42 & 0.30 \\
\hline 2715.01 & 11.128299 & 1.85 & 1052.062093 & 54.55 & 8.127 & 0.579 & 2.6784 & 8.280 & 0.56 & 0.27 \\
\hline 2715.02 & 2.226489 & 0.52 & 1066.274948 & 75.48 & 4.334 & 0.366 & 1.8312 & 6.696 & 0.43 & 0.30 \\
\hline 2715.03 & 5.720880 & 2.22 & 1067.165009 & 135.79 & 4.017 & 0.204 & 2.4888 & 7.848 & 0.44 & 0.31 \\
\hline 2764.01 & 2.252974 & 0.58 & 970.347138 & 91.65 & 2.146 & 0.176 & 1.7280 & 7.056 & 0.47 & 0.33 \\
\hline 2793.01 & 4.496868 & 0.91 & 1159.490499 & 53.06 & 4.541 & 0.262 & 1.7712 & 4.464 & 0.43 & 0.30 \\
\hline
\end{tabular}


Table 1

(Continued)

\begin{tabular}{|c|c|c|c|c|c|c|c|c|c|c|}
\hline KOI & $\begin{array}{c}P \\
\text { (days) }\end{array}$ & $\begin{array}{l}\delta P \\
(\mathrm{~s})\end{array}$ & $\begin{array}{c}t_{0} \\
\text { BJD-2454833 }\end{array}$ & $\begin{array}{l}\delta t_{0} \\
(\mathrm{~s})\end{array}$ & $\begin{array}{c}R_{p} / R_{\star} \\
(\%)\end{array}$ & $\begin{array}{c}\delta R_{p} / R_{\star} \\
(\%)\end{array}$ & $\begin{array}{l}\tau_{\text {tot }} \\
(\mathrm{hr})\end{array}$ & $\begin{array}{c}\delta \tau_{\text {tot }} \\
\text { (minutes) }\end{array}$ & $b$ & $\delta b$ \\
\hline 2793.02 & 1.766790 & 0.52 & 1163.121258 & 80.97 & 3.134 & 0.213 & 1.4088 & 5.112 & 0.47 & 0.33 \\
\hline 2839.01 & 2.164573 & 0.59 & 954.368569 & 98.60 & 2.202 & 0.161 & 1.3512 & 6.480 & 0.48 & 0.33 \\
\hline 2842.01 & 1.565414 & 0.15 & 1111.295284 & 29.66 & 5.349 & 0.465 & 0.8136 & 3.600 & 0.45 & 0.32 \\
\hline 2842.02 & 5.148931 & 1.10 & 1111.615116 & 67.33 & 4.999 & 0.428 & 0.9672 & 5.400 & 0.47 & 0.33 \\
\hline 2842.03 & 3.036220 & 0.60 & 1108.684748 & 60.98 & 4.362 & 0.477 & 0.9840 & 5.184 & 0.46 & 0.33 \\
\hline 2845.01 & 1.574091 & 0.40 & 860.664033 & 111.13 & 1.519 & 0.114 & 1.5720 & 6.624 & 0.48 & 0.33 \\
\hline 2862.01 & 24.575351 & 12.26 & 979.739313 & 179.76 & 2.938 & 0.253 & 2.2104 & 11.160 & 0.45 & 0.32 \\
\hline 2926.01 & 12.285498 & 7.52 & 1151.595094 & 162.99 & 4.138 & 0.221 & 3.0240 & 9.144 & 0.45 & 0.32 \\
\hline 2926.02 & 5.536076 & 2.53 & 1161.594913 & 125.53 & 3.513 & 0.197 & 2.0880 & 7.992 & 0.46 & 0.32 \\
\hline 2926.03 & 20.956929 & 14.59 & 1165.968351 & 195.86 & 4.371 & 0.281 & 3.6384 & 12.600 & 0.47 & 0.32 \\
\hline 2926.04 & 37.634156 & 64.86 & 1212.535073 & 386.23 & 3.936 & 0.241 & 4.4088 & 17.064 & 0.45 & 0.30 \\
\hline 2992.01 & 82.659402 & 73.12 & 813.954445 & 283.72 & 3.618 & 0.538 & 3.8616 & 22.824 & 0.50 & 0.36 \\
\hline 3010.01 & 60.866573 & 61.58 & 909.679601 & 375.88 & 2.850 & 0.236 & 4.7664 & 22.824 & 0.49 & 0.33 \\
\hline 3034.01 & 31.020889 & 18.05 & 851.497786 & 195.00 & 2.836 & 0.260 & 1.8744 & 11.664 & 0.45 & 0.33 \\
\hline 3094.01 & 4.577003 & 0.93 & 859.843063 & 89.46 & 2.494 & 0.249 & 0.9528 & 5.832 & 0.48 & 0.33 \\
\hline 3102.01 & 9.326378 & 7.39 & 855.995807 & 315.73 & 1.735 & 0.191 & 2.0472 & 14.688 & 0.47 & 0.33 \\
\hline 3119.01 & 2.184432 & 0.59 & 1066.429336 & 82.53 & 4.129 & 0.357 & 1.1352 & 5.904 & 0.45 & 0.32 \\
\hline 3140.01 & 5.688796 & 3.74 & 859.832880 & 254.73 & 1.478 & 0.134 & 2.7576 & 13.536 & 0.45 & 0.32 \\
\hline 3144.01 & 8.073945 & 4.10 & 1048.697851 & 186.12 & 3.132 & 0.220 & 2.1888 & 9.792 & 0.45 & 0.32 \\
\hline 3263.01 & 76.879365 & 4.69 & 761.917120 & 22.49 & 14.917 & 3.046 & 2.3928 & 5.760 & 0.68 & 0.29 \\
\hline 3282.01 & 49.276798 & 31.03 & 846.901733 & 269.07 & 3.575 & 0.292 & 3.7872 & 18.000 & 0.48 & 0.33 \\
\hline 3284.01 & 35.233209 & 22.62 & 840.028855 & 328.10 & 1.879 & 0.194 & 3.8328 & 20.160 & 0.43 & 0.31 \\
\hline 3414.01 & 27.009809 & 0.29 & 897.564195 & 3.69 & 33.676 & 4.891 & 2.0016 & 2.520 & 0.79 & 0.09 \\
\hline 3444.01 & 12.671432 & 7.50 & 859.778161 & 244.73 & 1.254 & 0.115 & 2.4624 & 12.456 & 0.45 & 0.32 \\
\hline 3444.02 & 60.326669 & 4.09 & 868.978630 & 30.12 & 4.568 & 0.524 & 1.5168 & 4.104 & 0.33 & 0.25 \\
\hline 3444.03 & 2.635964 & 1.19 & 859.838096 & 195.82 & 0.872 & 0.088 & 1.5816 & 10.008 & 0.46 & 0.32 \\
\hline 3444.04 & 14.150370 & 8.32 & 848.976183 & 260.63 & 1.212 & 0.139 & 1.6656 & 12.888 & 0.47 & 0.33 \\
\hline 3497.01 & 20.359756 & 5.95 & 846.882413 & 137.23 & 1.677 & 0.146 & 1.9320 & 9.432 & 0.47 & 0.33 \\
\hline 3749.01 & 10.727244 & 3.98 & 851.628048 & 12.96 & 33.302 & 7.451 & 1.8216 & 4.176 & 0.86 & 0.13 \\
\hline 4087.01 & 101.111336 & 74.93 & 923.296793 & 294.74 & 2.948 & 0.136 & 8.0304 & 16.560 & 0.45 & 0.31 \\
\hline 4252.01 & 15.571357 & 7.85 & 852.870658 & 213.79 & 1.199 & 0.122 & 2.1600 & 12.672 & 0.43 & 0.31 \\
\hline 4290.01 & 4.838142 & 5.92 & 725.405466 & 114.25 & 4.610 & 0.360 & 1.2768 & 7.128 & 0.45 & 0.31 \\
\hline 4427.01 & 147.661348 & 110.51 & 982.258277 & 382.50 & 3.205 & 0.257 & 6.1056 & 20.160 & 0.44 & 0.30 \\
\hline 4875.01 & 0.912184 & 0.49 & 861.553074 & 207.01 & 1.211 & 0.142 & 1.1280 & 11.232 & 0.47 & 0.33 \\
\hline 5228.01 & 546.280708 & 5544.62 & 880.611075 & 4355.96 & 2.483 & 0.413 & 33.9576 & 302.760 & 0.52 & 0.36 \\
\hline 5359.01 & 2.719979 & 15.24 & 584.861009 & 257.65 & 2.783 & 0.255 & 2.0352 & 12.312 & 0.46 & 0.33 \\
\hline 5692.01 & 2.641814 & 1.62 & 861.675662 & 244.83 & 0.754 & 0.071 & 2.3232 & 13.752 & 0.45 & 0.32 \\
\hline
\end{tabular}

Note.

${ }^{a}$ Transit parameters derived from fits to individual transit times. Period and mid-transit time are used from fits assuming linear ephemeris.

detrended. We reject outliers from the phase folded transit signal by binning the data into bins that are one half the integration time of the observations or with widths that contain at least 20 data points per bin. From the distribution of data points in each bin, a robust estimation of the standard deviation is calculated using the median absolute deviation:

$$
\operatorname{MAD}=\operatorname{median}\left(\left|x_{i}-\operatorname{median}(\boldsymbol{x})\right|\right),
$$

where the residuals are given by $\boldsymbol{x}=\left\{x_{0}, x_{1} \ldots x_{n}\right\}$. MAD is then scaled to estimate the standard deviation assuming a Gaussian distribution so that $\sigma=1.4826 \mathrm{MAD}$, and then data are rejected with absolute deviation from the median beyond a threshold $n \sigma$, where

$$
n=\sqrt{2} \operatorname{erf}^{-1}(1-\eta / N),
$$

and where $N$ is the number of data point under consideration. Removing outliers in this manner produces a minimal effect on the statistical properties of the data by removing points that are inconsistent with the original robust estimation of the standard deviation of the sample given the sample size. We use a value of $\eta=0.1$ which translates to $2.8 \lesssim n \lesssim 4.0$ for our data set.

\section{TRANSIT FITTING}

\subsection{Long and Short Cadence Fits Using a Linear Ephemeris Model}

We characterize our vetted sample of 163 planet candidates around 104 cool stars by first fitting all of the long and short cadence data available with a linear ephemeris transit model using a Markov Chain Monte Carlo parameter estimation algorithm. Our light curve model uses the analytic solutions from Mandel \& Agol (2002) for a quadratic stellar limb darkening law that provides a relative flux model for planet-tostar size ratio, projected separation, and limb darkening parameters. The hyper-geometric functions of those solutions need to be evaluated numerically and present a computational barrier. We therefore use a circular planet orbit to convert time 


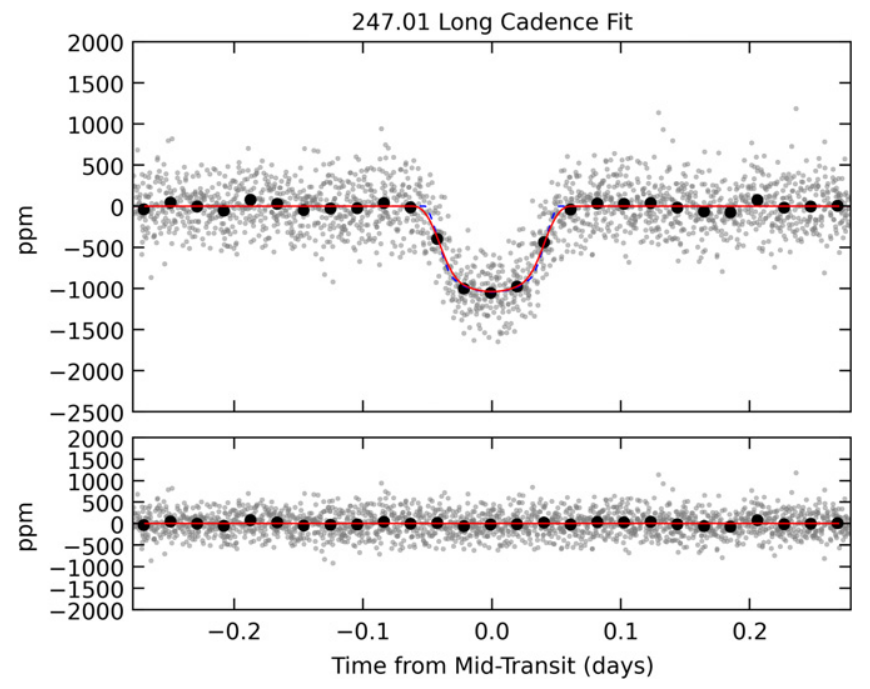

Figure 3. Phase folded long cadence data for KOI-247.01 are shown as gray dots. These data binned at a timescale approximately equal to the original sampling of the long cadence data stream are shown as black dots for viewing purposes only. The best-fit model is shown in red and the residuals of this fit are shown in the bottom panel. The raw model (without resampling) is shown as a blue dashed line for reference.

(The complete figure set (163 images) is available.)

into projected separation for a given period and transit duration. This allows us to side-step solving Kepler's equation, and instead perform the transformation from time to relative separation between the star and planet with simple trigonometric functions. Under this approximation, the ingress and egress of the model are exactly symmetric, also halving the number of computations needed for each model call. Of course, this does not allow for subtle effects due to eccentric orbits to be adequately modeled and care must be taken when interpreting the derived transit duration in terms of stellar density (Seager \& Mallén-Ornelas 2003; Kipping 2010b). However, for our sample, this effect can be accounted for and is expected to have a negligible effect on the derived transit parameters.

We parametrize our model with the scaled planet radius, $R_{p} /$ $R_{\star}$; the impact parameter, $b$; the duration from the first to the fourth contact point of the transit, $\tau_{\text {tot }}$; the time of mid-transit as measured nearest to the middle of the Kepler light curve, $t_{0}$; the period, $P$; and two limb darkening parameters, $q_{1}$ and $q_{2}$, which characterize the full range of quadratic parameter space of monotonically decreasing and positive value profiles (Kipping 2013).

Before our models can be compared to data, the effect of finite integration times must be considered (e.g., Kipping 2010a; Price \& Rogers 2014). The Kepler Mission produced time series data sampled at two different intervals using a single exposure time. The exposure time (accumulated time of flux from a celestial source on a given pixel) is $t_{\exp }=6.020 \mathrm{~s}$, and for every exposure there is a fixed CCD readout time of $t_{\text {read }}=0.519 \mathrm{~s}$. The short cadence data is made up of nine such exposures and therefore the time between the start of successive short cadence data is $\left(t_{\exp }+t_{\text {read }}\right) \times 9=58.849 \mathrm{~s}$. However, the time interval over which the astronomical signal is integrated is one read shorter than this, i.e., $t_{\text {smooth }}^{\text {short }}=9 t_{\text {exp }}+8 t_{\text {read }}=58.330 \mathrm{~s}$. Similarly, the long cadence data are made up of 270 integrations and therefore the time between successive integration times is $t_{\text {cadence }}^{\text {long }}=1765.463 \mathrm{~s}$ and the smoothing time $t_{\text {smooth }}^{\text {long }}=1764.944 \mathrm{~s}$.

To account for the effects of integration time, we first calculate the planet path across the stellar disk assuming that the planet is in a circular orbit using $b=a \cos (i) / R_{\star}$. The light curve for this planetary trajectory is oversampled and then smoothed using a uniform filter of width $t_{\text {smooth. This is }}$ analogous to the resampling procedure recommended by Kipping (2010a), and we hereafter refer to this process as resampling. The degree of resampling needed to produce an accurate model using this method will depend on the transit parameters. Therefore, we numerically determine the optimal resampling for each transit candidate based on the parameters from preliminary fits enforcing an resampling of at least five. For a grid of transit parameter values spanning the full range of $R_{p} / R_{\star}$ and $\tau_{\text {tot }}$ in our data set, and for an impact parameter of 0 (the effect of finite integration time is most severe for low impact parameter transits), we first calculate a reference transit model resampled by a factor of 3001 . We then calculate transit curves for the same set of input parameters resampled in steps of 2 from 3 to 501. The smallest resampling value that produces peak-to-peak discrepancies with the reference model of less than one part per million is then recorded. We then construct a grid of values from this procedure that we use to interpolate the optimal resampling values to be used for any of our targets based on their preliminary transit parameters.

We use a Bayesian framework to determine the best fit values for our seven model parameters and their associated errors. To evaluate the likelihood, we do not resample the model at each data timestamp. Instead, we phase fold the data at each trial period, $P$, and mid-transit time, $t_{0}$, and interpolate our resampled model to the phase folded timestamps of the data. This speeds up each likelihood call by an order of magnitude or more. The quantity $\left(R_{p} / R_{\star}\right)^{2}$ is a scale parameter in the problem and we therefore apply a Jefferys prior to this parameter. We note that this has a small to negligible effect on our posterior samples as we are data-dominated rather than prior-dominated for the majority of our transit candidates. Each of the other free parameters have uniform priors (i.e., no prior).

We use the emcee affine invariant Markov Chain Monte Carlo ensemble sampler (Foreman-Mackey et al. 2013) with 1000 chains, or "walkers" $\left(n_{w}=1000\right)$. The initial values of each walker were over-dispersed in most parameters based on the estimated values found by fitting the transit shape with a quick and flexible Levenberg-Marquardt fitting algorithm (Markwardt 2009). The relative planet radius, $R_{p} / R_{\star}$, is dispersed in a uniform manner from 0 to a factor of 2 larger than the value obtained from the preliminary fit; the full duration, $\tau_{\text {tot }}$, is dispersed from half to twice the preliminary fit value; the impact parameter, $b$, is dispersed uniformly from 0 to 1 ; the period, $P$, is dispersed by $\pm 1 \mathrm{~s}$ from the nominal value; the mid-transit times, $t_{0}$, uniformly span 2 minutes; and the limb darkening parameters, $q_{1}$ and $q_{2}$, are uniformly dispersed between 0 and 1 .

The walkers are evolved for $n_{b}=1000$ steps and then analyzed. We use the correlation length, $c_{l}$, to assess if the chains have reached a sufficiently mixed state. The burn-in stage was re-run with a larger number of steps if the number of independent draws, $n_{b} n_{w} / c_{l}$, was found to be less than 10,000 . The sampler was then reset and the walkers restarted from their last location for an additional 1000 steps. These last 1000 steps 
Table 2

Transit Parameters for Short Cadence Fits

\begin{tabular}{|c|c|c|c|c|c|c|c|c|c|c|}
\hline KOI & $\begin{array}{c}P \\
\text { (days) } \\
\end{array}$ & $\begin{array}{l}\delta P \\
(\mathrm{~s})\end{array}$ & $\begin{array}{c}t_{0} \\
\text { BJD-2454833 }\end{array}$ & $\begin{array}{l}\delta t_{0} \\
(\mathrm{~s})\end{array}$ & $\begin{array}{c}R_{p} / R_{\star} \\
\% \\
\end{array}$ & $\begin{array}{c}\delta R_{p} / R_{\star} \\
\% \\
\end{array}$ & $\begin{array}{l}\tau_{\text {tot }} \\
(\mathrm{hr})\end{array}$ & $\begin{array}{c}\delta \tau_{\text {tot }} \\
\text { (minutes) }\end{array}$ & $b$ & $\delta b$ \\
\hline 247.01 & 13.815048 & 2.79 & 816.617079 & 35.00 & 2.932 & 0.094 & 2.0760 & 2.016 & 0.36 & 0.24 \\
\hline $248.01^{\mathrm{a}}$ & 7.203856 & 0.68 & 1063.565400 & 27.16 & 4.511 & 0.128 & 2.7024 & 2.448 & 0.82 & 0.03 \\
\hline $248.02^{\mathrm{a}}$ & 10.912775 & 2.26 & 1064.699387 & 66.43 & 3.142 & 0.099 & 2.8056 & 2.016 & 0.24 & 0.18 \\
\hline 248.03 & 2.576568 & 0.18 & 1063.620781 & 23.68 & 2.711 & 0.135 & 1.6512 & 1.800 & 0.48 & 0.26 \\
\hline 248.04 & 18.596035 & 5.66 & 1071.069820 & 98.67 & 2.631 & 0.217 & 2.3592 & 5.904 & 0.47 & 0.31 \\
\hline 249.01 & 9.549278 & 0.75 & 853.755275 & 13.74 & 3.949 & 0.106 & 1.5936 & 1.080 & 0.29 & 0.20 \\
\hline $250.01^{\mathrm{a}}$ & 12.282930 & 1.08 & 1067.057517 & 25.08 & 5.526 & 0.207 & 2.9400 & 2.664 & 0.81 & 0.05 \\
\hline $250.02^{\mathrm{a}}$ & 17.251180 & 2.64 & 1046.949926 & 45.84 & 4.825 & 0.438 & 2.1336 & 4.824 & 0.76 & 0.12 \\
\hline 250.03 & 3.543901 & 0.81 & 1064.763415 & 73.09 & 1.960 & 0.187 & 2.0280 & 4.680 & 0.52 & 0.32 \\
\hline 250.04 & 46.827619 & 11.22 & 1073.299935 & 90.27 & 3.905 & 0.493 & 2.0064 & 7.272 & 0.72 & 0.26 \\
\hline 251.01 & 4.164381 & 0.15 & 1049.773828 & 9.61 & 4.658 & 0.145 & 1.7976 & 1.080 & 0.37 & 0.20 \\
\hline 251.02 & 5.774490 & 4.41 & 1045.321872 & 139.32 & 1.549 & 0.116 & 1.7184 & 7.992 & 0.48 & 0.33 \\
\hline 252.01 & 17.604678 & 2.32 & 1033.124115 & 35.64 & 4.429 & 0.160 & 3.5328 & 2.808 & 0.35 & 0.24 \\
\hline 253.01 & 6.383154 & 0.54 & 1057.864519 & 24.52 & 4.446 & 0.257 & 1.7712 & 2.304 & 0.61 & 0.22 \\
\hline 253.02 & 20.617213 & 26.45 & 1073.333911 & 423.48 & 2.210 & 0.227 & 3.8496 & 21.384 & 0.46 & 0.33 \\
\hline 254.01 & 2.455241 & 0.01 & 1064.529313 & 1.62 & 19.021 & 0.384 & 1.8192 & 0.504 & 0.53 & 0.01 \\
\hline 255.01 & 27.521998 & 3.59 & 1043.004555 & 38.58 & 4.561 & 0.188 & 4.1160 & 3.096 & 0.39 & 0.24 \\
\hline 255.02 & 13.603507 & 22.57 & 1051.814672 & 442.25 & 1.275 & 0.110 & 3.1488 & 14.976 & 0.48 & 0.33 \\
\hline $314.01^{\mathrm{a}}$ & 13.781059 & 0.71 & 1059.843180 & 14.34 & 2.603 & 0.101 & 2.3064 & 1.440 & 0.63 & 0.13 \\
\hline $314.02^{\mathrm{a}}$ & 23.089024 & 2.74 & 1048.389953 & 41.93 & 2.413 & 0.394 & 1.9296 & 5.616 & 0.68 & 0.31 \\
\hline $314.03^{\mathrm{a}}$ & 10.313760 & 5.69 & 1072.004278 & 182.45 & 1.026 & 0.098 & 1.8240 & 8.064 & 0.39 & 0.28 \\
\hline $463.01^{\mathrm{a}}$ & 18.477200 & 12.32 & 1367.835784 & 40.59 & 5.046 & 0.198 & 1.8216 & 2.520 & 0.35 & 0.24 \\
\hline 531.01 & 3.687460 & 0.54 & 904.686698 & 7.11 & 6.278 & 1.089 & 1.0848 & 1.944 & 0.56 & 0.27 \\
\hline 571.01 & 7.267344 & 1.12 & 1104.529642 & 45.95 & 2.533 & 0.125 & 2.2560 & 2.736 & 0.49 & 0.29 \\
\hline 571.02 & 13.342947 & 2.38 & 1110.913718 & 48.25 & 2.727 & 0.076 & 2.7720 & 3.024 & 0.66 & 0.23 \\
\hline 571.03 & 3.886790 & 0.38 & 1108.913123 & 31.08 & 2.107 & 0.087 & 1.8504 & 1.728 & 0.45 & 0.28 \\
\hline 571.04 & 22.407795 & 5.32 & 1117.331057 & 88.11 & 2.423 & 0.110 & 3.2640 & 4.032 & 0.40 & 0.28 \\
\hline 571.05 & 129.944005 & 339.37 & 1086.446411 & 900.93 & 2.147 & 0.181 & 5.2536 & 28.872 & 0.47 & 0.32 \\
\hline 596.01 & 1.682697 & 0.26 & 865.405753 & 24.09 & 2.598 & 0.140 & 1.4112 & 1.944 & 0.57 & 0.25 \\
\hline 739.01 & 1.287078 & 0.26 & 903.448937 & 30.66 & 2.578 & 0.147 & 1.5144 & 2.304 & 0.51 & 0.30 \\
\hline 812.01 & 3.340224 & 0.47 & 1200.766635 & 32.11 & 3.942 & 0.143 & 1.9080 & 2.304 & 0.39 & 0.27 \\
\hline 812.02 & 20.060078 & 15.04 & 1190.605490 & 176.60 & 3.692 & 0.319 & 3.5040 & 10.008 & 0.45 & 0.32 \\
\hline 812.03 & 46.184101 & 46.30 & 1181.286403 & 222.49 & 3.609 & 0.215 & 4.9728 & 12.384 & 0.44 & 0.30 \\
\hline 812.04 & 7.825101 & 6.01 & 1185.104652 & 183.34 & 2.157 & 0.174 & 2.3784 & 8.712 & 0.46 & 0.32 \\
\hline 817.01 & 23.967393 & 39.23 & 1288.749229 & 281.41 & 3.353 & 0.438 & 4.0176 & 17.496 & 0.55 & 0.34 \\
\hline 817.02 & 8.295646 & 5.09 & 1288.883486 & 103.50 & 2.730 & 0.200 & 1.1976 & 5.184 & 0.46 & 0.32 \\
\hline 854.01 & 56.053787 & 99.52 & 1266.237042 & 280.44 & 4.142 & 0.246 & 4.7304 & 11.952 & 0.48 & 0.30 \\
\hline $886.01^{\mathrm{a}}$ & 8.009985 & 9.59 & 1347.809468 & 135.95 & 3.812 & 0.203 & 2.4336 & 7.200 & 0.84 & 0.14 \\
\hline $886.02^{\mathrm{a}}$ & 12.072588 & 31.23 & 1350.556846 & 284.08 & 3.184 & 0.172 & 3.0240 & 5.040 & 0.39 & 0.27 \\
\hline 886.03 & 20.995881 & 56.93 & 1328.082217 & 345.37 & 2.717 & 0.215 & 3.0432 & 16.632 & 0.50 & 0.33 \\
\hline $898.01^{\mathrm{a}}$ & 9.770428 & 1.74 & 1113.680872 & 43.37 & 4.344 & 0.115 & 2.4552 & 2.088 & 0.40 & 0.25 \\
\hline 898.02 & 5.169829 & 0.94 & 1113.536547 & 59.64 & 3.078 & 0.138 & 2.2584 & 2.736 & 0.37 & 0.26 \\
\hline 898.03 & 20.090228 & 10.10 & 1112.306231 & 132.13 & 3.549 & 0.275 & 3.8400 & 9.000 & 0.57 & 0.31 \\
\hline 899.01 & 7.113708 & 1.56 & 1184.472277 & 53.03 & 2.686 & 0.155 & 2.1840 & 3.168 & 0.50 & 0.28 \\
\hline 899.02 & 3.306547 & 0.65 & 1182.547053 & 40.07 & 2.159 & 0.109 & 1.7544 & 2.304 & 0.47 & 0.34 \\
\hline 899.03 & 15.368448 & 5.23 & 1177.078002 & 78.63 & 2.687 & 0.148 & 2.4432 & 4.176 & 0.46 & 0.30 \\
\hline 936.01 & 9.467874 & 3.26 & 954.776076 & 27.44 & 4.564 & 0.110 & 2.4624 & 1.728 & 0.26 & 0.18 \\
\hline 936.02 & 0.893039 & 0.17 & 953.458458 & 18.75 & 2.588 & 0.109 & 1.1256 & 1.152 & 0.50 & 0.27 \\
\hline 947.01 & 28.598917 & 18.95 & 1276.691772 & 85.81 & 3.881 & 0.175 & 3.7704 & 6.552 & 0.51 & 0.26 \\
\hline 952.01 & 5.901300 & 1.55 & 1162.819235 & 65.71 & 4.135 & 0.507 & 2.4096 & 6.480 & 0.71 & 0.26 \\
\hline $952.02^{\mathrm{a}}$ & 8.751986 & 3.96 & 1168.372434 & 111.13 & 3.927 & 0.240 & 2.5008 & 8.136 & 0.85 & 0.14 \\
\hline 952.03 & 22.780779 & 7.94 & 1180.338720 & 75.57 & 4.436 & 0.134 & 3.3168 & 5.760 & 0.48 & 0.28 \\
\hline 952.04 & 2.896003 & 1.47 & 1164.593353 & 106.21 & 2.008 & 0.165 & 1.7448 & 5.400 & 0.46 & 0.32 \\
\hline 952.05 & 0.742960 & 0.25 & 1163.018720 & 83.96 & 1.551 & 0.117 & 1.1520 & 3.960 & 0.51 & 0.33 \\
\hline 961.01 & 1.213771 & 0.02 & 1199.760924 & 4.12 & 4.229 & 0.116 & 0.5424 & 0.360 & 0.41 & 0.21 \\
\hline 961.02 & 0.453287 & 0.01 & 1199.548330 & 2.97 & 4.133 & 0.128 & 0.4488 & 0.288 & 0.46 & 0.20 \\
\hline 961.03 & 1.865111 & 0.05 & 1200.637140 & 6.53 & 3.613 & 0.123 & 0.4512 & 0.504 & 0.44 & 0.28 \\
\hline 1078.01 & 3.353711 & 0.56 & 1201.355442 & 47.99 & 3.615 & 0.186 & 1.5840 & 3.240 & 0.47 & 0.33 \\
\hline 1078.02 & 6.877478 & 1.41 & 1198.090516 & 62.73 & 4.031 & 0.215 & 1.2576 & 2.808 & 0.43 & 0.29 \\
\hline 1078.03 & 28.464390 & 18.41 & 1182.769700 & 179.24 & 3.765 & 0.227 & 2.6784 & 7.848 & 0.43 & 0.31 \\
\hline 1201.01 & 2.757584 & 4.90 & 954.809350 & 129.03 & 2.298 & 0.277 & 1.0368 & 6.912 & 0.48 & 0.34 \\
\hline 1408.01 & 14.534993 & 29.40 & 1337.221964 & 439.96 & 2.046 & 0.198 & 2.8776 & 19.296 & 0.47 & 0.33 \\
\hline 1725.01 & 9.878617 & 1.63 & 1412.757288 & 13.37 & 3.698 & 0.126 & 1.9248 & 1.368 & 0.39 & 0.21 \\
\hline
\end{tabular}


Table 2

(Continued)

\begin{tabular}{|c|c|c|c|c|c|c|c|c|c|c|}
\hline KOI & $\begin{array}{c}P \\
\text { (days) }\end{array}$ & $\begin{array}{l}\delta P \\
(\mathrm{~s})\end{array}$ & $\begin{array}{c}t_{0} \\
\text { BJD-2454833 }\end{array}$ & $\begin{array}{l}\delta t_{0} \\
(\mathrm{~s})\end{array}$ & $\begin{array}{c}R_{p} / R_{\star} \\
\%\end{array}$ & $\begin{array}{c}\delta R_{p} / R_{\star} \\
\%\end{array}$ & $\begin{array}{l}\tau_{\text {tot }} \\
(\mathrm{hr})\end{array}$ & $\begin{array}{c}\delta \tau_{\text {tot }} \\
(\text { minutes })\end{array}$ & $b$ & $\delta b$ \\
\hline 1843.01 & 4.194588 & 1.98 & 1329.565344 & 55.83 & 2.409 & 0.206 & 1.8768 & 3.960 & 0.47 & 0.32 \\
\hline $1843.02^{\mathrm{b}}$ & 6.355921 & 33.60 & 1332.399317 & 1539.87 & 0.002 & 0.008 & 6.1416 & 574.128 & 0.50 & 0.34 \\
\hline 1867.01 & 2.549561 & 1.57 & 1346.199599 & 62.28 & 2.282 & 0.236 & 1.7880 & 5.040 & 0.74 & 0.28 \\
\hline 1867.02 & 13.969476 & 8.67 & 1333.548100 & 76.73 & 3.196 & 0.335 & 1.3968 & 4.824 & 0.49 & 0.33 \\
\hline 1867.03 & 5.212287 & 3.18 & 1347.174910 & 78.00 & 2.139 & 0.099 & 2.1480 & 3.960 & 0.46 & 0.33 \\
\hline 2036.01 & 8.410908 & 45.64 & 1521.619841 & 258.10 & 2.885 & 0.286 & 2.1720 & 11.376 & 0.47 & 0.32 \\
\hline $2036.02^{b}$ & 5.795352 & 33.41 & 1516.076576 & 3536.86 & 0.000 & 0.001 & 20.6520 & 1105.416 & 0.50 & 0.34 \\
\hline 2418.01 & 86.806562 & 496.84 & 1404.881538 & 1138.05 & 2.811 & 0.382 & 6.7512 & 57.816 & 0.49 & 0.34 \\
\hline 2650.01 & 34.987138 & 95.73 & 1368.842232 & 475.54 & 2.088 & 0.895 & 4.2984 & 20.088 & 0.48 & 0.33 \\
\hline 2650.02 & 7.054275 & 22.60 & 1342.482081 & 404.28 & 1.777 & 0.244 & 1.7520 & 16.272 & 0.48 & 0.33 \\
\hline 2704.01 & 4.869934 & 121.13 & 1575.526914 & 225.74 & 22.127 & 2.903 & 2.0088 & 15.840 & 0.49 & 0.33 \\
\hline 2704.02 & 2.983711 & 161.90 & 1574.470943 & 525.50 & 14.312 & 17.881 & 1.7064 & 39.456 & 0.56 & 0.36 \\
\hline $2704.03^{b}$ & 8.176432 & 4321.04 & 1569.090938 & 1618.76 & 0.000 & 0.000 & 45.5976 & 1875.672 & 0.50 & 0.33 \\
\hline 2842.01 & 1.565443 & 7.63 & 1574.656534 & 57.51 & 5.535 & 0.415 & 0.7728 & 2.736 & 0.44 & 0.30 \\
\hline 2842.02 & 5.149013 & 28.07 & 1575.020298 & 120.44 & 5.349 & 0.601 & 0.8496 & 5.544 & 0.45 & 0.32 \\
\hline 2842.03 & 3.036239 & 16.96 & 1576.259874 & 232.30 & 4.132 & 2.533 & 1.0056 & 12.456 & 0.47 & 0.33 \\
\hline
\end{tabular}

Notes.

a Transit parameters derived from fits to individual transit times. Period and mid-transit time are used from fits assuming linear ephemeris.

$\mathrm{b}$ Transit fit failed due to insufficient data.

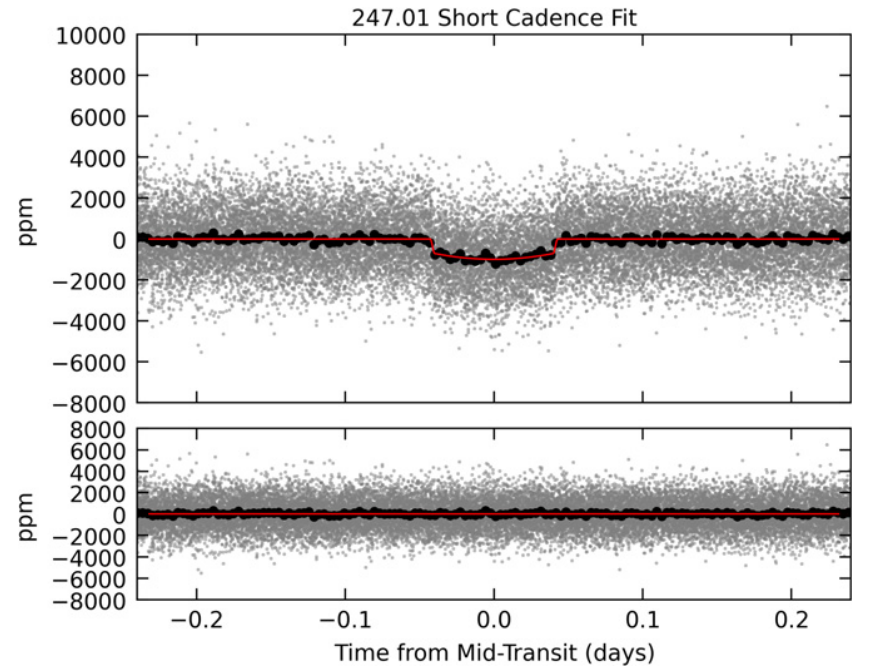

Figure 4. Same as Figure 3, but for the KOI-247.01 short cadence data.

(The complete figure set (79 images) is available.)

for each 1000 walkers $\left(10^{6}\right.$ samples total $)$ comprise the final posterior samples that we use to estimate the transit parameters.

The results of the long cadence data fits are summarized in Table 1, and an example fit can be seen in Figure 3. A fit to the short cadence data for this same KOI can be seen in Figure 4. The median values for planet period, mid-transit time, relative radius, duration, and impact parameter are reported along with the half width of the shortest $1 \sigma$ interval of the posterior for each parameter. These values are a useful reference. However, they conceal details about the probability of the these parameter values. Figure 5 shows a series of the two-dimensional posterior probability distributions for the seven free parameters in the fits. The expected covariance between parameters such as the impact parameter, $b$, and the relative size of the planet, $R_{p} / R_{\star}$, can be clearly seen. The MCMC chains are available for download such that these parameter dependencies can be properly accounted for in future statistical studies.

For 79 transit signals toward $36 \mathrm{cool}$ KOIs there exist short cadence data. We follow the exact procedure outlined above for these data including preliminary fits and MCMC posterior sampling. These results are summarized in Table 2. The short cadence data fit for the same KOI shown in Figure 3, KOI247.01, is shown in Figure 4 for reference. We note that the short cadence MCMC fits for KOI-1843.02, KOI-2036.03, and KOI-2704.03 failed due primarily to lack of sufficient data. These fits are included for completeness. However, the results from the long cadence fits should be used for further study.

\subsection{Transit Timing Variations}

\subsubsection{TTV Search}

For each transit signal, we use the best-fitting transit model to fit for the times of each transit event in search of potential TTVs (Agol et al. 2005; Holman \& Murray 2005). A single parameter, $\Delta t_{0}$, quantifies the time deviation of mid-transit in relation to the expected time based on a linear ephemeris from the best fits. The model light curve is fit to each transit event letting only $\Delta t_{0}$ float using a Levenberg-Marquardt minimization (Markwardt 2009) to produce a list of observed-minuscalculated $(O-C)$ values corresponding to each transit. Figure 6 shows an example of one of the known TTV planets in our sample, KOI-248.01.

To assess the significance of potential TTV signals, we first calculate the rms scatter in the times of mid-transit as estimated by the median absolute deviation $\sigma_{O-C}$ and compare that to the median value of the estimated errors on the transit times $\bar{\sigma}_{\mathrm{TT}}$ (Mazeh et al. 2013). We consider values of $\sigma_{O}-C / \bar{\sigma}_{\mathrm{TT}}>3.0$ to be significant. Next, we compute a Lomb Normalized Periodogram $^{9}$ for the calculated $O-C$ transit times. We calculate a $p$ value for this peak by producing 10,000

\footnotetext{
http://www.exelisvis.com/docs/LNP_TEST.html
} 


\subsection{Long Cadence Fit Posterior Distributions}

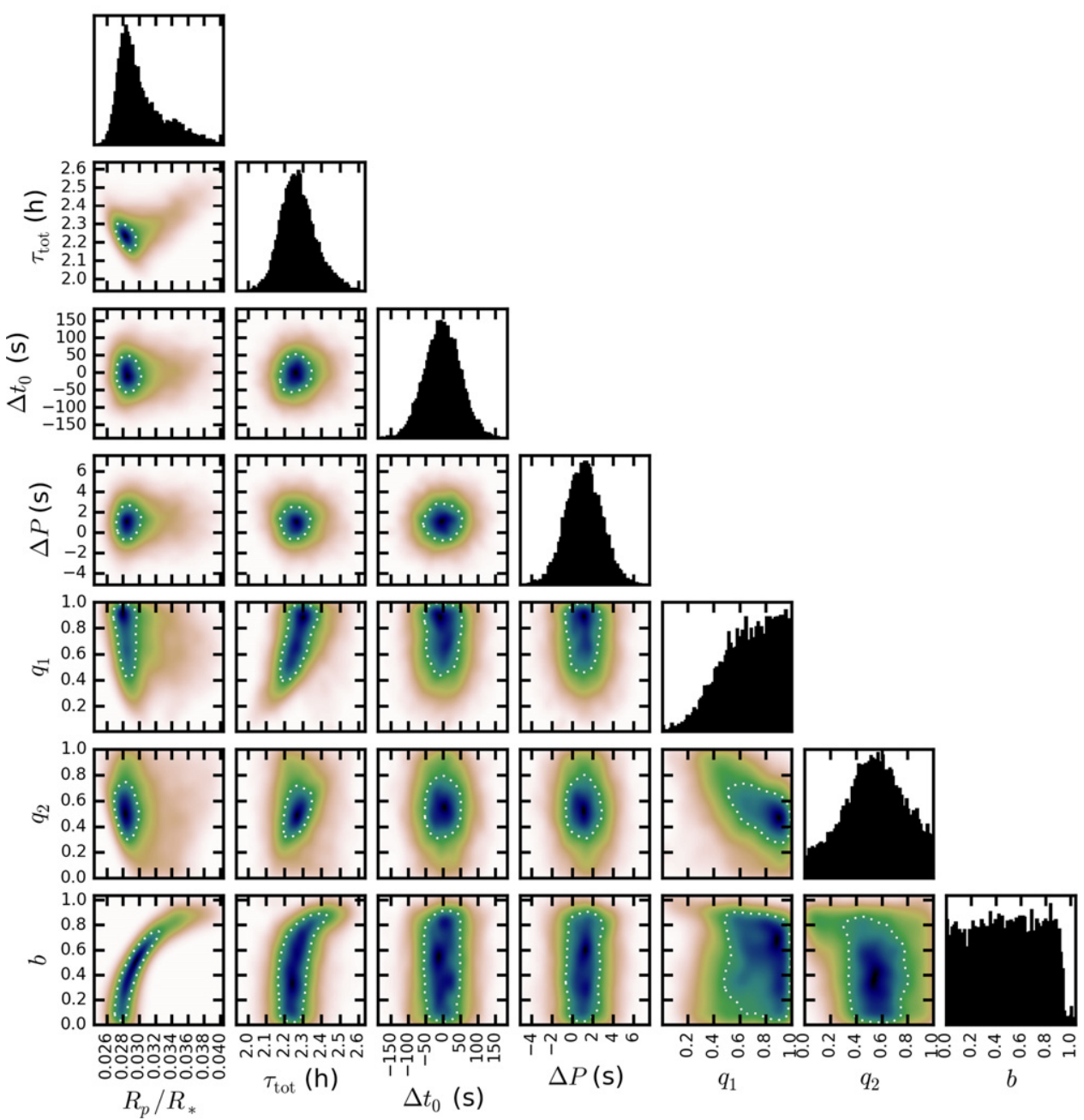

Figure 5. Array of $1 \mathrm{D}$ and 2D posteriors for the long cadence fit shown in Figure 3. The 2D posteriors were constructed using a 2D kernel density estimation that reveals covariances between parameters, most notably for $R_{p} / R_{\star}, b$, and $\tau_{\text {tot }}$. The $68.3 \%$ confidence contour for each 2D posterior is designated with a dotted white line.

(The complete figure set (163 images) is available.)

periodograms for the $O-C$ data randomly scrambled. The fractional number of periodogram peaks in the simulation that are greater than or equal to the original peak is interpreted as the probability that the measured periodogram is due to random noise, $p_{\text {LNP. }}$ This probability value is considered to be significant when $p_{\mathrm{LNP}} \leqslant 0.001$.

Finally, we fit both a sine curve and a polynomial to the $O-C$ data. The sine curve model contains an amplitude, period, phase, and offset. The starting parameters for the fit are a one minute amplitude, a period equal to the location of the peak of the periodogram, and zero phase and offset. To assess the significance of the fit results for the polynomial and sine curve models, we perform an $\mathrm{F}$ test on the fitted parameters by comparing the $\chi^{2}$ values and degrees of freedom from a single parameter fit (a mean) and the polynomial or sine model. We again consider $p_{\text {sine }} \leqslant 0.001$ and $p_{\text {poly }} \leqslant 0.001$ to be significant.

\subsubsection{TTV Results}

The results were scrutinized by eye to weed out TTV signals due to stroboscopic effects and other, non-dynamical processes
(Szabó et al. 2013). The results from our TTV search are summarized in Table 3 . We recover $12 \mathrm{KOIs}$ with significant TTV signals, 11 of which are in multi-transiting systems. These 12 planet candidates comprise $7.4 \%$ of the full $\mathrm{M}$ dwarf planet candidate sample and are found toward 7 of the 104, or $6.7 \%$ of all M dwarf KOIs. All of our TTV detections have been detected previously and are reported in the literature $(\mathrm{Wu} \&$ Lithwick 2013; Mazeh et al. 2013; Kipping et al. 2014). However, these new transit timing results use only data from the Kepler mission. Following are a few notes regarding our TTV search.

KOI-3284 is reported to have a significant TTV signal by Kipping et al. (2014). Our tests show a signal at a period of 190 days in both the periodogram and the sinusoid fit. However, the false alarm probability of the periodogram peak is found to be very high and this KOI also failed our F test for the sinusoidal fit. Therefore, we do not include this planet candidate in our list. KOI-2306 has $\sigma_{O-C} / \sigma_{\mathrm{TT}}^{-}=3.12$ due to the under sampling of the transit by the long cadence data, and we therefore exclude it. KOI-1907 and KOI-2130 show some signs of long period TTV signals at $\sim 700$ and $\sim 1100$ day 
periods, respectively. However, both of these signals fall narrowly below our selection criteria and are therefore excluded.

KOI-952.02 is not reported by Mazeh et al. (2013) as a significant TTV source. However, we find that in 17 quarters of data the periodicity at $\sim 260$ days is significant. This matches the period reported by Fabrycky et al. (2012). KOI-952.01 does not produce a signal significant enough to warrant inclusion in our list, although we do find that the first eight quarters of data are consistent with the results of Fabrycky et al. (2012), and a period of 260 days is apparent in our periodogram as the second highest peak but with a high formal false positive probability (FPP).

\subsubsection{Fitting Transit Signals with TTVs}

Transit timing variations can significantly affect the perceived transit shape under the assumption of a linear ephemeris. The effect essentially smears out the ingress and

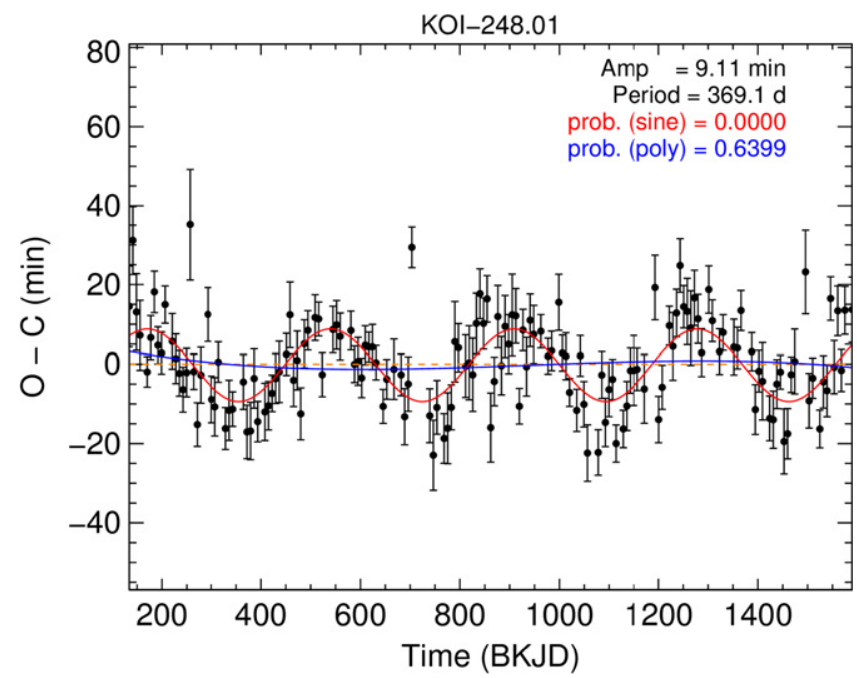

Figure 6. Transit timing variations $(O-C)$ of KOI-248.01 fit with a pure sinusoid (red) and a polynomial (blue). These fits are only used to assess the significance of a potential TTV signal and are not used to fit the transits (see Section 4.2.3).

(The complete figure set (12 images) is available.) egress and potentially fills in the depth of the transit. The details depend on the exact nature of the TTVs. However, typically TTVs will bias the impact parameter to higher values, the transit duration to larger values, and the limb darkening parameters will tend toward values that produce a more severe contrast between the center of the star and the limb.

Due to these effects, we refit the transit signals in our sample that show significant TTVs after folding on the individual transit times derived above. We first reject any individual transits that have mid-transit time errors that are either ill defined or more than $2 \sigma$ from the median error. We then perform a transit fit using the same model outlined in Section 4, except that instead of fitting the period and mid-transit time, we fix the individual transit times.

We choose a large TTV source, KOI-886.01, as an example showing the potential effects of fitting a linear transit model to a planet that displays significant TTVs. The $\sim 2 \mathrm{hr}$ peak-to-peak TTVs for KOI-886.01 bias the fits toward a larger impact parameter, a smaller planet, and a longer duration. The median posterior values for the impact parameter and relative planet size are discrepant at the 0.3 and $1.2 \sigma$ levels. However the derived transit durations are in disagreement with $98 \%$ confidence. These results are shown in Figure 7.

\section{FALSE POSITIVE PROBABILITY}

The Kepler pipeline is known to have produced a highfidelity sample of transiting exoplanets (Wu et al. 2010; Morton \& Johnson 2011; Morton 2012; Christiansen et al. 2013; Fressin et al. 2013). Up to this point, we have treated every signal as a transiting exoplanet. However, it is prudent to assign to each transit signal a probability that the signal was generated from another astrophysical scenario. We use the methods of Morton \& Johnson (2011) and Morton (2012) to analyze the light curves shapes that we have extracted to assign an FPP of each transit signal independently.

These FPPs are reported in Table 4 along with the probability of the transiting planet scenario compared to all other astrophysical scenarios, $P=L_{\mathrm{TP}} / L_{\mathrm{FP}}$; the specific occurrence assumed in the calculation, $f_{\mathrm{pl} \text {,specific }}$; and the specific planet occurrence needed to achieve a threshold FPP of 0.005 , $f_{p, V}$. Included in each calculation is also a confusion radius within which false positives are permitted to exist. For this

Table 3

M Dwarf Planets with Transit Timing Variations

\begin{tabular}{|c|c|c|c|c|c|c|c|c|}
\hline KOI & $N$ & $\sigma_{O-C} / \bar{\sigma}_{\mathrm{TT}}$ & LNP Amp. & $p_{\mathrm{LNP}}$ & $\begin{array}{c}\text { Sine Amp. } \\
\text { (minutes) }\end{array}$ & $\begin{array}{c}P_{\mathrm{TTV}} \\
(\text { days })\end{array}$ & $p_{\text {sine }}$ & $p_{\text {poly }}$ \\
\hline 248.01 & 159 & 2.19 & 29.15 & 0.0001 & 9.71 & 365.97 & 0.00000 & 0.68916 \\
\hline 250.01 & 95 & 1.86 & 22.62 & 0.0001 & 9.76 & 743.74 & 0.00000 & 0.03606 \\
\hline 250.02 & 52 & 1.63 & 9.38 & 0.0009 & 7.60 & 809.02 & 0.00007 & 0.18420 \\
\hline 314.01 & 71 & 1.85 & 12.29 & 0.0001 & 5.46 & 1111.01 & 0.00000 & 0.00000 \\
\hline 314.02 & 50 & 1.88 & 14.91 & 0.0001 & 13.86 & 1022.36 & 0.00000 & 0.00000 \\
\hline 886.01 & 158 & 1.82 & 43.24 & 0.0001 & 57.79 & 818.59 & 0.00000 & 0.06058 \\
\hline 886.02 & 99 & 1.66 & 22.35 & 0.0001 & 105.95 & 871.50 & 0.00000 & 0.00706 \\
\hline 898.01 & 123 & 0.89 & 7.37 & 0.0579 & 7.37 & 334.12 & 0.00060 & 0.86390 \\
\hline 952.02 & 103 & 1.15 & 11.27 & 0.0001 & 17.61 & 261.62 & 0.00012 & 0.13167 \\
\hline
\end{tabular}

Notes. Entries in boldface denote statistically significant values in our search for TTV signals (see text). 


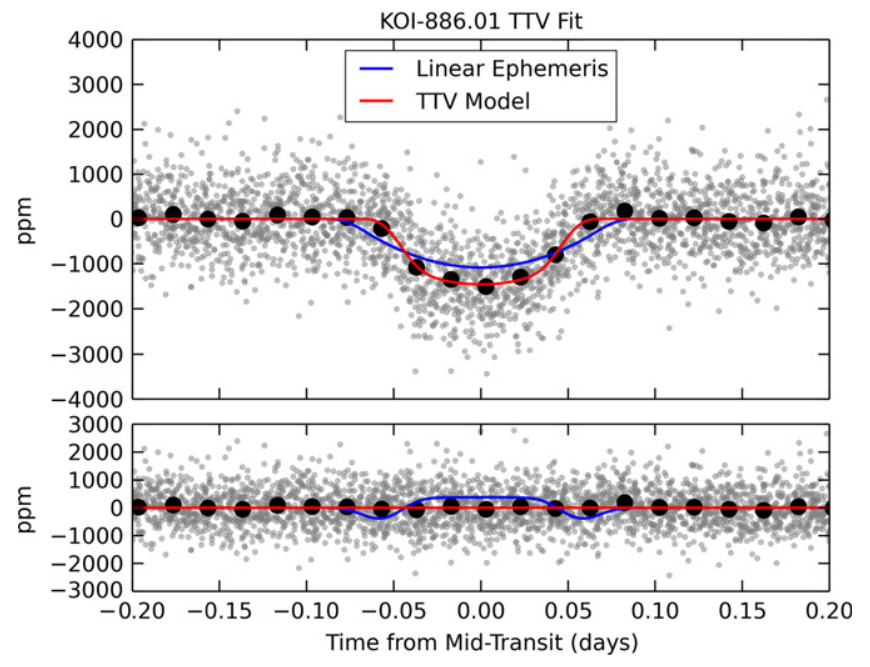

Figure 7. (Top) Long cadence Kepler photometry of KOI-886.01 phase folded on the transit times derived in Section 4.2. The best-fit model assuming a linear ephemeris is shown in blue and the best-fit model for the data folded on the non-linear transit times is shown in red. (Bottom) The residuals of the best-fit non-linear model. The difference between the linear and non-linear models is shown in blue. Assuming a linear ephemeris for this target which shows peakto-peak TTVs of $\sim 2 \mathrm{hr}$ significantly affects the derived transit parameters, in particular, the transit duration.

radius, we use three times the uncertainty in the multi-quarter difference-image pixel response function (PRF) fit reported in the Exoplanet Archive [the "PRF $\Delta \theta_{\mathrm{MQ}}$ (OOT)" column]. The minimum exclusion radius we allow is 0.5 arcsec, and the default value we use if no value is available is 4 arcsec. An example of a diagnostic plot generated by the FPP analysis is shown in Figure 8.

We find that $11 \%$ of the sample, or 18 of the 163 , has a FPP of larger than $10 \%$, consistent with estimates for the entire Kepler sample (Morton \& Johnson 2011; Fressin et al. 2013). However, six of these high FPP targets are either known planets in the literature (e.g., KOI-254.01, Johnson et al. 2011b; KOI-886.02, Steffen et al. 2013; and KOI-1422.05, Rowe et al. 2014) or are part of three or four transit systems much less likely to be a false positives. Therefore, this is a high-fidelity sample of transiting exoplanets around the lowestmass stars observed by the Kepler primary mission.

We do note that our treatment of exclusion radius ignores the possibility of more distant PRF contamination, as detected via the period-epoch match study of Coughlin et al. (2014), which found that "parent" eclipsing stars even up to 10-100 arcsec from the target star were able to cause "child" false positive signals. While that work discovered over 600 false positive KOIs, it also highlighted the possibility of further distant contaminants that might remain undetected because the "parent" may not be a known eclipsing system.

In order to estimate the rough probability of any of the present KOIs being false positives via this mechanism, we can use the numbers discussed by Coughlin et al. (2014). That work identified $12 \%$ of all known KOIs (not all planet candidates) to be due to PRF contamination. However, they pointed out that only about $1 / 3$ of the stars in the Kepler field were downloaded, and so it might be reasonable to assume that for every discovered PRF contaminant, there might be two undiscovered, bringing the overall rate to about $36 \%$. According to this reasoning, about $24 \%$ of all KOIs might be PRF contaminants that cannot be discovered by the periodepoch match method.

Table 4

False Positive Probability Results

\begin{tabular}{|c|c|c|c|c|}
\hline KOI & FPP & $P$ & $f_{p, \text { specific }}$ & $f_{p, V}$ \\
\hline 247.01 & 0.0165 & 215 & 0.276 & 0.92400 \\
\hline $248.01^{\mathrm{a} b}$ & 0.0000 & 290321 & 0.218 & 0.00069 \\
\hline $248.02^{\mathrm{a} b}$ & 0.0902 & 39 & 0.258 & 5.08000 \\
\hline $248.03^{\mathrm{b}}$ & 0.0004 & 8541 & 0.276 & 0.02330 \\
\hline $248.04^{\mathrm{b}}$ & 0.0018 & 1998 & 0.276 & 0.09960 \\
\hline 249.01 & 0.0000 & 848734 & 0.269 & 0.00023 \\
\hline $250.01^{\mathrm{a} \mathrm{b}}$ & 0.0940 & 64 & 0.149 & 3.08000 \\
\hline $250.02^{\mathrm{a} b}$ & 0.0069 & 707 & 0.202 & 0.28200 \\
\hline $250.03^{\mathrm{b}}$ & 0.0003 & 10975 & 0.276 & 0.01820 \\
\hline $250.04^{\mathrm{b}}$ & 0.0030 & 1432 & 0.229 & 0.13900 \\
\hline $251.01^{\mathrm{b}}$ & 0.0003 & 17497 & 0.195 & 0.01140 \\
\hline $251.02^{\mathrm{b}}$ & 0.0036 & 997 & 0.276 & 0.20000 \\
\hline 252.01 & 0.0018 & 2685 & 0.202 & 0.07400 \\
\hline $253.01^{\mathrm{b}}$ & 0.0122 & 428 & 0.189 & 0.46500 \\
\hline $253.02^{\mathrm{b}}$ & 0.0012 & 3041 & 0.276 & 0.06570 \\
\hline 254.01 & 0.3690 & 171 & 0.010 & 1.20000 \\
\hline $255.01^{\mathrm{b}}$ & 0.0011 & 4614 & 0.195 & 0.04320 \\
\hline $255.02^{\mathrm{b}}$ & 0.0213 & 166 & 0.276 & 1.20000 \\
\hline $314.01^{\mathrm{a} \mathrm{b}}$ & 0.0000 & 162471 & 0.276 & 0.00123 \\
\hline $314.02^{\mathrm{a} b}$ & 0.0000 & 86675 & 0.276 & 0.00230 \\
\hline $314.03^{\mathrm{a} b}$ & 0.0069 & 520 & 0.276 & 0.38300 \\
\hline $463.01^{\mathrm{a}}$ & 0.0000 & 842598 & 0.276 & 0.00024 \\
\hline 478.01 & 0.0000 & 91416 & 0.226 & 0.00218 \\
\hline 531.01 & 0.4820 & 23 & 0.046 & 8.49000 \\
\hline $571.01^{\mathrm{b}}$ & 0.0002 & 14547 & 0.276 & 0.01370 \\
\hline $571.02^{\mathrm{b}}$ & 0.0000 & 241542 & 0.276 & 0.00082 \\
\hline $571.03^{\mathrm{b}}$ & 0.0000 & 88366 & 0.276 & 0.00226 \\
\hline $571.04^{\mathrm{b}}$ & 0.0014 & 2495 & 0.276 & 0.08000 \\
\hline $571.05^{\mathrm{b}}$ & 0.0046 & 787 & 0.276 & 0.25300 \\
\hline 596.01 & 0.0000 & 355210 & 0.276 & 0.00056 \\
\hline 641.01 & 0.0026 & 1406 & 0.276 & 0.14200 \\
\hline 739.01 & 0.0001 & 54976 & 0.276 & 0.00363 \\
\hline 781.01 & 0.0104 & 511 & 0.186 & 0.39000 \\
\hline $812.01^{\mathrm{b}}$ & 0.0000 & 117784 & 0.227 & 0.00169 \\
\hline $812.02^{\mathrm{b}}$ & 0.0001 & 64936 & 0.238 & 0.00307 \\
\hline $812.03^{\mathrm{b}}$ & 0.0001 & 50413 & 0.241 & 0.00395 \\
\hline $812.04^{\mathrm{b}}$ & 0.0098 & 366 & 0.276 & 0.54400 \\
\hline $817.01^{\mathrm{b}}$ & 0.0003 & 15136 & 0.258 & 0.01320 \\
\hline $817.02^{\mathrm{b}}$ & 0.0136 & 262 & 0.276 & 0.75700 \\
\hline 818.01 & 0.0001 & 29391 & 0.233 & 0.00677 \\
\hline 854.01 & 0.0002 & 22853 & 0.250 & 0.00871 \\
\hline $886.01^{\mathrm{a} b}$ & 0.0269 & 136 & 0.265 & 1.46000 \\
\hline $886.02^{\mathrm{a} b}$ & 1.0000 & 0 & 0.276 & Inf \\
\hline $886.03^{\mathrm{b}}$ & 0.0109 & 328 & 0.276 & 0.60700 \\
\hline $898.01^{\mathrm{a} b}$ & 0.0001 & 49290 & 0.207 & 0.00404 \\
\hline $898.02^{\mathrm{b}}$ & 0.0000 & 154069 & 0.266 & 0.00129 \\
\hline $898.03^{\mathrm{b}}$ & 0.0004 & 11540 & 0.244 & 0.01720 \\
\hline $899.01^{\mathrm{b}}$ & 0.0000 & 128024 & 0.276 & 0.00155 \\
\hline $899.02^{\mathrm{b}}$ & 0.0001 & 32059 & 0.276 & 0.00622 \\
\hline $899.03^{\mathrm{b}}$ & 0.0001 & 38377 & 0.276 & 0.00519 \\
\hline $936.01^{\mathrm{b}}$ & 0.0000 & 412281 & 0.231 & 0.00048 \\
\hline $936.02^{\mathrm{b}}$ & 0.0000 & 126681 & 0.276 & 0.00157 \\
\hline 947.01 & 0.0000 & 578234 & 0.251 & 0.00034 \\
\hline $952.01^{\mathrm{b}}$ & 0.0006 & 6507 & 0.243 & 0.03060 \\
\hline $952.02^{\mathrm{a} b}$ & 0.0268 & 145 & 0.249 & 1.36000 \\
\hline $952.03^{\mathrm{b}}$ & 0.0012 & 3900 & 0.217 & 0.05100 \\
\hline $952.04^{\mathrm{b}}$ & 0.0141 & 253 & 0.276 & 0.78400 \\
\hline $952.05^{\mathrm{b}}$ & 0.0240 & 147 & 0.276 & 1.35000 \\
\hline $961.01^{\mathrm{b}}$ & 0.0119 & 300 & 0.276 & 0.66200 \\
\hline $961.02^{\mathrm{b}}$ & 0.0052 & 691 & 0.276 & 0.28800 \\
\hline $961.03^{\mathrm{b}}$ & 0.0424 & 81 & 0.276 & 2.44000 \\
\hline $1078.01^{\mathrm{b}}$ & 0.0000 & 102712 & 0.266 & 0.00194 \\
\hline $1078.02^{\mathrm{b}}$ & 0.0019 & 2155 & 0.249 & 0.09260 \\
\hline $1078.03^{\mathrm{b}}$ & 0.0046 & 881 & 0.247 & 0.22500 \\
\hline
\end{tabular}


Table 4

(Continued)

\begin{tabular}{|c|c|c|c|c|}
\hline KOI & FPP & $P$ & $f_{p, \text { specific }}$ & $f_{p, V}$ \\
\hline 1085.01 & 0.0011 & 3414 & 0.276 & 0.05830 \\
\hline 1141.01 & 0.0002 & 16390 & 0.276 & 0.01210 \\
\hline 1146.01 & 0.0028 & 1272 & 0.276 & 0.15700 \\
\hline 1201.01 & 0.0024 & 1499 & 0.276 & 0.13300 \\
\hline 1393.01 & 0.0150 & 290 & 0.226 & 0.68500 \\
\hline 1397.01 & 0.0058 & 712 & 0.242 & 0.28000 \\
\hline 1408.01 & 0.0025 & 1445 & 0.276 & 0.13800 \\
\hline $1422.01^{b}$ & 0.0000 & 118789 & 0.276 & 0.00168 \\
\hline $1422.02^{\mathrm{b}}$ & 0.0001 & 35517 & 0.276 & 0.00561 \\
\hline $1422.03^{b}$ & 0.0051 & 701 & 0.276 & 0.28400 \\
\hline $1422.04^{\mathrm{b}}$ & 0.0061 & 592 & 0.276 & 0.33600 \\
\hline $1422.05^{\mathrm{b}}$ & 0.1740 & 17 & 0.276 & 11.60000 \\
\hline 1427.01 & 0.0001 & 31230 & 0.276 & 0.00640 \\
\hline 1649.01 & 0.1800 & 16 & 0.276 & 12.10000 \\
\hline $1681.01^{\mathrm{b}}$ & 0.7360 & 1 & 0.276 & 153.00000 \\
\hline $1681.02^{b}$ & 0.0089 & 403 & 0.276 & 0.49300 \\
\hline $1681.03^{b}$ & 0.0182 & 195 & 0.276 & 1.02000 \\
\hline 1702.01 & 0.0073 & 491 & 0.276 & 0.40600 \\
\hline 1725.01 & 0.0007 & 5536 & 0.271 & 0.03590 \\
\hline $1843.01^{b}$ & 0.0181 & 196 & 0.276 & 1.01000 \\
\hline $1843.02^{b}$ & 0.0122 & 293 & 0.276 & 0.67700 \\
\hline $1867.01^{\mathrm{b}}$ & 0.0047 & 770 & 0.276 & 0.25900 \\
\hline $1867.02^{\mathrm{b}}$ & 0.0155 & 233 & 0.272 & 0.85500 \\
\hline $1867.03^{\mathrm{b}}$ & 0.0024 & 1493 & 0.276 & 0.13400 \\
\hline 1868.01 & 0.0020 & 1994 & 0.249 & 0.09990 \\
\hline 1879.01 & 0.0782 & 90 & 0.130 & 2.19000 \\
\hline 1880.01 & 0.0009 & 4071 & 0.276 & 0.04890 \\
\hline 1902.01 & 0.9340 & 0 & 0.254 & 719.00000 \\
\hline 1907.01 & 0.0005 & 8143 & 0.268 & 0.02440 \\
\hline 2006.01 & 0.0017 & 2153 & 0.276 & 0.09230 \\
\hline $2036.01^{\mathrm{b}}$ & 0.0336 & 104 & 0.276 & 1.91000 \\
\hline $2036.02^{\mathrm{b}}$ & 0.0215 & 164 & 0.276 & 1.21000 \\
\hline 2057.01 & 0.0086 & 419 & 0.276 & 0.47500 \\
\hline 2058.01 & 0.0032 & 1121 & 0.276 & 0.17700 \\
\hline 2090.01 & 0.0036 & 1043 & 0.266 & 0.19100 \\
\hline 2130.01 & 0.0045 & 846 & 0.262 & 0.23500 \\
\hline 2156.01 & 0.0732 & 48 & 0.260 & 4.08000 \\
\hline $2179.01^{b}$ & 0.0023 & 1592 & 0.270 & 0.12500 \\
\hline $2179.02^{\mathrm{b}}$ & 0.0281 & 125 & 0.276 & 1.59000 \\
\hline 2191.01 & 0.1180 & 27 & 0.276 & 7.38000 \\
\hline 2238.01 & 0.0069 & 522 & 0.276 & 0.38100 \\
\hline 2306.01 & 0.0107 & 334 & 0.276 & 0.59500 \\
\hline 2329.01 & 0.1120 & 28 & 0.276 & 6.93000 \\
\hline 2347.01 & 0.0063 & 572 & 0.276 & 0.34800 \\
\hline 2417.01 & 0.1860 & 15 & 0.276 & 12.50000 \\
\hline 2418.01 & 0.0125 & 286 & 0.276 & 0.69600 \\
\hline 2453.01 & 0.0267 & 132 & 0.276 & 1.51000 \\
\hline 2480.01 & 0.0878 & 37 & 0.276 & 5.30000 \\
\hline 2542.01 & 0.0079 & 455 & 0.276 & 0.43700 \\
\hline 2626.01 & 0.0392 & 88 & 0.276 & 2.24000 \\
\hline $2650.01^{\mathrm{b}}$ & 0.0072 & 501 & 0.276 & 0.39700 \\
\hline $2650.02^{\mathrm{b}}$ & 0.0703 & 47 & 0.276 & 4.16000 \\
\hline 2662.01 & 0.0071 & 504 & 0.276 & 0.39500 \\
\hline $2704.01^{b}$ & 0.0011 & 5920 & 0.148 & 0.03360 \\
\hline $2704.02^{b}$ & 0.0014 & 2814 & 0.259 & 0.07030 \\
\hline $2704.03^{\mathrm{b}}$ & 0.9600 & 0 & 0.276 & 1320.00000 \\
\hline 2705.01 & 0.0001 & 26834 & 0.276 & 0.00741 \\
\hline $2715.01^{b}$ & 0.0562 & 218 & 0.077 & 0.91700 \\
\hline $2715.02^{b}$ & 0.0089 & 486 & 0.228 & 0.40900 \\
\hline $2715.03^{b}$ & 0.0071 & 572 & 0.244 & 0.34700 \\
\hline 2764.01 & 0.0005 & 6583 & 0.276 & 0.03030 \\
\hline $2793.01^{\mathrm{b}}$ & 0.0009 & 4685 & 0.232 & 0.04250 \\
\hline $2793.02^{\mathrm{b}}$ & 0.0076 & 475 & 0.276 & 0.41900 \\
\hline 2839.01 & 0.0011 & 3382 & 0.276 & 0.05890 \\
\hline
\end{tabular}

Table 4

(Continued)

\begin{tabular}{lcccc}
\hline \hline KOI & FPP & $P$ & $f_{p, \text { specific }}$ & $f_{p, V}$ \\
\hline $2842.01^{\mathrm{b}}$ & 0.0001 & 32059 & 0.276 & 0.00622 \\
$2842.02^{\mathrm{b}}$ & 0.0177 & 201 & 0.276 & 0.99100 \\
$2842.03^{\mathrm{b}}$ & 0.0005 & 6977 & 0.276 & 0.02850 \\
2845.01 & 0.0002 & 19268 & 0.276 & 0.01030 \\
2862.01 & 0.0193 & 184 & 0.275 & 1.07000 \\
$2926.01^{\mathrm{b}}$ & 0.0005 & 10656 & 0.193 & 0.01860 \\
$2926.02^{\mathrm{b}}$ & 0.0014 & 3188 & 0.232 & 0.06250 \\
$2926.03^{\mathrm{b}}$ & 0.0049 & 1145 & 0.178 & 0.17400 \\
$2926.04^{\mathrm{b}}$ & 0.0004 & 11417 & 0.206 & 0.01740 \\
2992.01 & $\mathbf{0 . 3 4 6 0}$ & 8 & 0.233 & 24.60000 \\
3010.01 & 0.0026 & 1422 & 0.275 & 0.14000 \\
3034.01 & 0.0039 & 935 & 0.276 & 0.21300 \\
3094.01 & 0.0183 & 194 & 0.276 & 1.02000 \\
3102.01 & 0.0214 & 165 & 0.276 & 1.20000 \\
3119.01 & 0.0014 & 2495 & 0.276 & 0.07980 \\
3140.01 & $\mathbf{0 . 2 6 2 0}$ & 10 & 0.276 & 19.60000 \\
3144.01 & 0.0007 & 5553 & 0.276 & 0.03590 \\
3263.01 & $\mathbf{0 . 7 1 4 0}$ & 16 & 0.024 & 11.80000 \\
3282.01 & 0.0008 & 4860 & 0.243 & 0.04090 \\
3284.01 & 0.0078 & 457 & 0.276 & 0.43500 \\
3414.01 & $\mathbf{0 . 9 6 2 0}$ & 13 & 0.003 & 17.30000 \\
$3444.01^{\mathrm{b}}$ & 0.0046 & 777 & 0.276 & 0.25600 \\
$3444.02^{\mathrm{b}}$ & $\mathbf{0 . 4 1 3 0}$ & 6 & 0.211 & 29.60000 \\
$3444.03^{\mathrm{b}}$ & 0.0370 & 94 & 0.276 & 2.11000 \\
$3444.04^{\mathrm{b}}$ & 0.0416 & 83 & 0.276 & 2.38000 \\
3497.01 & 0.0001 & 31230 & 0.276 & 0.00637 \\
3749.01 & $\mathbf{0 . 8 5 5 0}$ & 14 & 0.012 & 14.30000 \\
4087.01 & 0.0004 & 8442 & 0.276 & 0.02360 \\
4252.01 & 0.0124 & 288 & 0.276 & 0.69000 \\
4290.01 & 0.0238 & 148 & 0.276 & 1.34000 \\
4427.01 & 0.0636 & 54 & 0.268 & 3.62000 \\
4875.01 & 0.0022 & 1635 & 0.276 & 0.12200 \\
5228.01 & $\mathbf{0 . 8 5 3 0}$ & 0 & 0.276 & 320.00000 \\
5359.01 & 0.0006 & 6109 & 0.274 & 0.03260 \\
5692.01 & 0.0124 & 288 & 0.276 & 0.68900 \\
\hline & & & & \\
\hline
\end{tabular}

Notes. Entries in boldface denote False positive probabilities larger than $10 \%$. These values are derived without consideration of the presence of TTV signals or other transit signals toward the same source.

${ }^{\text {a }}$ Source of significant TTV signal.

${ }^{\mathrm{b}}$ Multi-transit candidate system.

However, they also go on to point out that $5 / 6$ of the false positives they detected were also identified as false positives by other methods (e.g., pixel-centroid offsets, detected secondary eclipses, etc). So this implies that of those previously mentioned $24 \%$, only $1 / 6$ of those, or $4 \%$ of all KOIs, may be long-distance PRF contaminants undetected by any Kepler FP vetting procedure and thus achieving planet candidate status. Comparing this to the $\sim 64 \%$ of all KOIs expected to be true planets, we estimate that an additional $\sim 6-7 \%$ of Kepler planet candidates, beyond what we calculate here using the methods of Morton (2012), could still be false positives. Incorporating in detail this additional long-distance PRF contamination into quantitative models of false positive probability is thus warranted but beyond the scope of this present work.

In addition, we also note that the FPPs presented in this paper do not consider the number of independent transit signals in the light curve or the possibility of detected TTVs, both of 
K00247.01

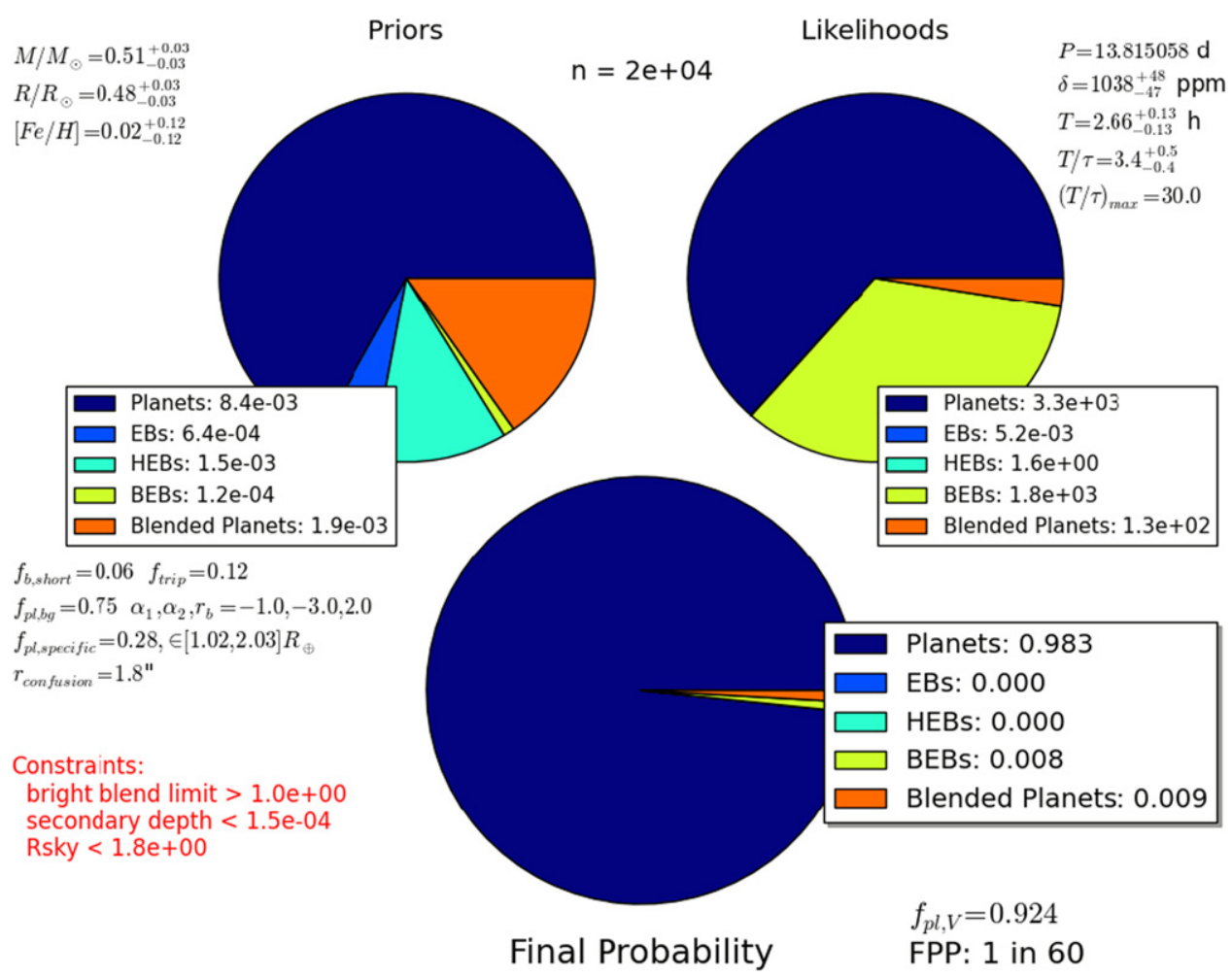

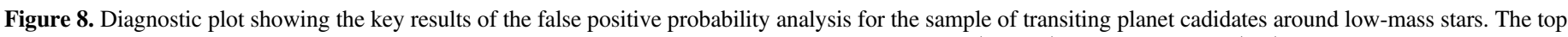

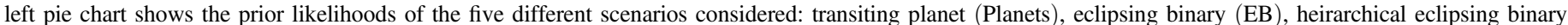

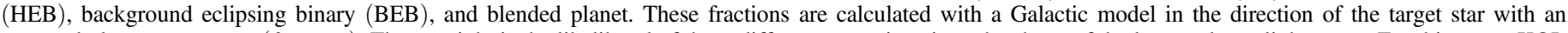

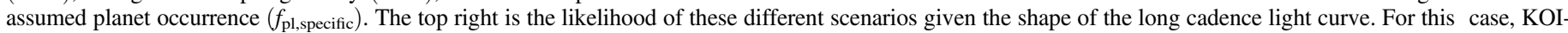

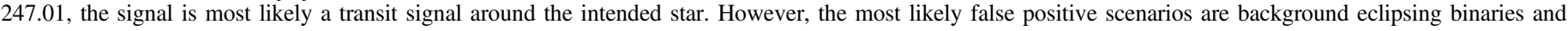
blended planet signals.

(The complete figure set (163 images) is available.)

which may substantially reduce the FPP (e.g., Ford et al. 2011; Lissauer et al. 2014; Rowe et al. 2014).

\section{THE ENSEMBLE OF M DWARF PLANET CANDIDATES}

The cool KOI catalog enables the study of the smallest and possibly most numerous planet population discovered by Kepler and helps to advance our knowledge of planet formation around the most common types of stars. It is estimated that $75 \%$ of the stars within $10 \mathrm{pc}$ are M dwarfs (Henry et al. 1994, 2004; Reid \& Cruz 2002). Therefore, by targeting this population we are also learning what can be expected of the closest planetary systems outside our solar system.

To further our understanding of this sample of small planets, we present uniformly derived transit parameters for all known transit signals around cool KOIs. These stars constitute a small fraction (about 2\%) of the total Kepler targets. However, the sample is large enough to allow for meaningful statistical analyses (Ballard \& Johnson 2014; Morton \& Swift 2014). Since M dwarf stars are difficult to characterize observationally, it is also important that our sample be small enough such that each individual star can be addressed with followup observations.

The planet candidates of this work have been drawn from the Exoplanet Archive list using the cool dwarf photometric cuts of
Mann et al. (2012). Additional vetting was performed using near-infrared, medium-resolution spectroscopy (Muirhead et al. 2012b, 2014). Our final sample contains 163 planets around $104 \mathrm{cool}$ stars. The total number of single transit systems is 74 ; meanwhile, there are 12 double systems, 10 triple systems, 5 quadruple systems, and 3 quintuple systems. A total of $54.6 \%$ of these planets are found in multi-transit systems, and $12.4 \%$ of these multis show significant TTV signals. On the contrary, only one single transit system out of 74 , or $1.4 \%$, shows a significant TTV signal.

The final results of our transit fits to the Kepler long and short cadence data are summarized in Tables 1 and 2, respectively. These tables display the results from the linear ephemeris model for all KOIs except those listed in Table 3. For those sources we report the period, $P$, and mid-transit time, $t_{0}$, from the linear ephemeris fits (although it should be noted that these parameters are not strictly defined in this context) and the other transit parameters from the non-linear ephemeris fits. An earlier version of this catalog has already been used in the literature to infer the statistical properties of the Kepler M dwarf planet population (Morton \& Swift 2014), and is presented here so that it may be used for further statistical studies. Each transit signal has been treated individually, and we have generated posterior samples of the seven transit parameters using uninformed priors that are available for download along with a suite of diagnostic plots for each KOI. 


\section{Cumulative Transit Parameter Distributions}
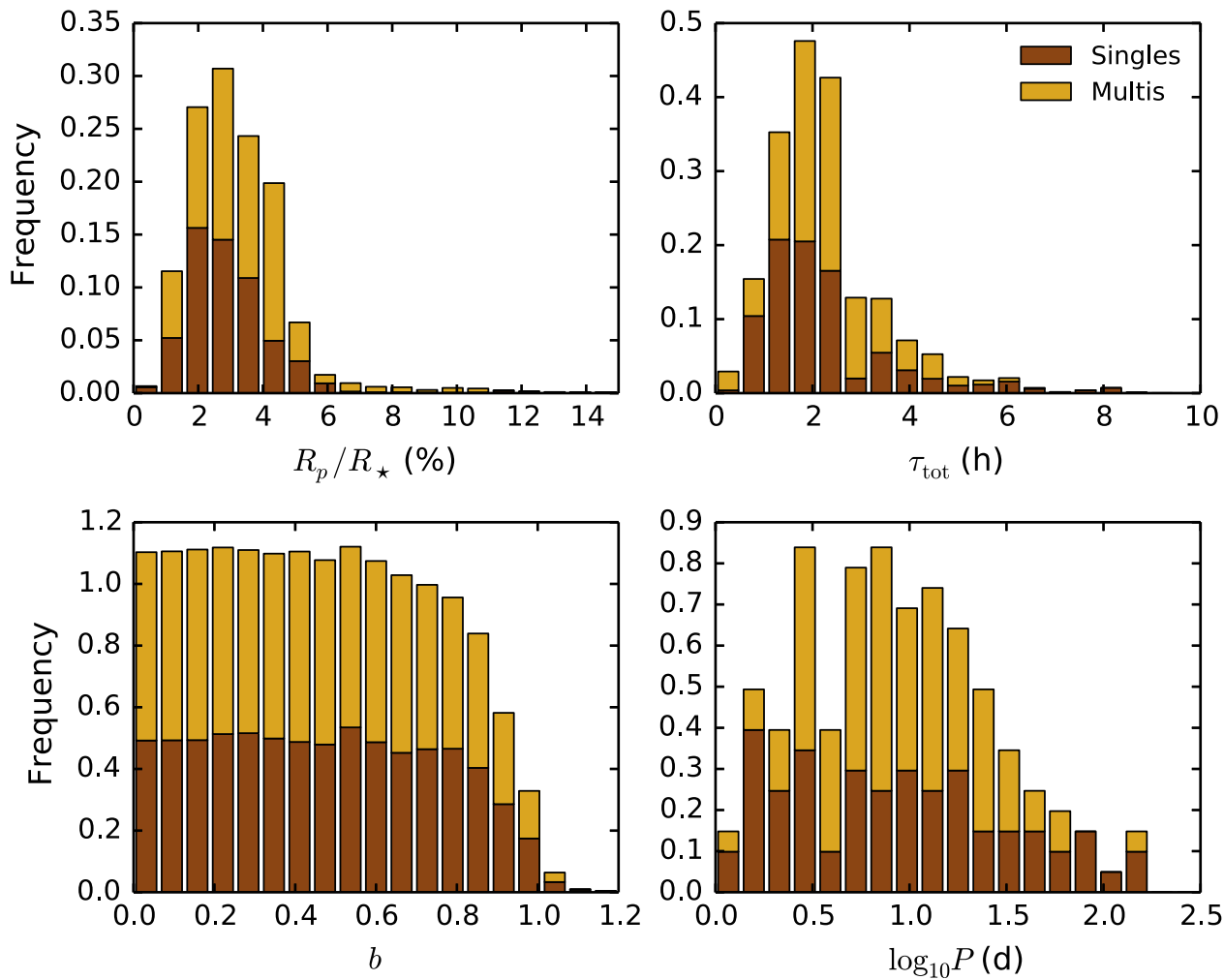

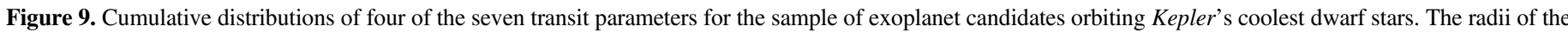

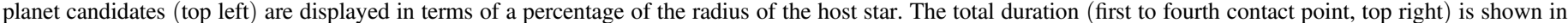

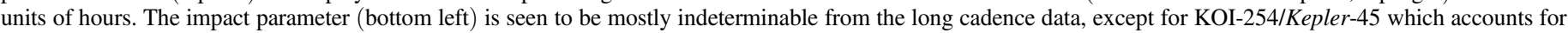

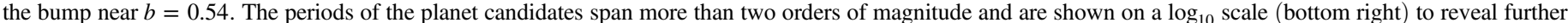
details of the distribution. The stacked histograms differentiate the sample of single transit systems (brown) and planets in multi-transit systems (gold).

Table 5

M Star Kepler Objects of Interest

\begin{tabular}{|c|c|c|c|c|c|c|c|c|c|c|c|}
\hline KOI & KIC & $N_{\mathrm{pl}}$ & $\operatorname{Mass}\left(M_{\odot}\right)$ & Radius $\left(R_{\odot}\right)$ & $T_{\text {eff }}(\mathrm{K})$ & {$[\mathrm{Fe} / \mathrm{H}](\mathrm{dex})$} & Ref. & $P_{\text {rot }}($ days $)$ & $K_{p}$ & $J$ & $K_{s}$ \\
\hline 247 & 11852982 & 1 & $0.51_{-0.03}^{+0.03}$ & $0.48_{-0.03}^{+0.03}$ & $3735_{-33}^{+49}$ & $0.02_{-0.12}^{+0.12}$ & 1 & 16.2 & 14.22 & 12.01 & 11.12 \\
\hline 248 & 5364071 & 4 & $0.55_{-0.04}^{+0.04}$ & $0.52_{-0.04}^{+0.04}$ & $3838_{-74}^{+111}$ & $-0.02_{-0.14}^{+0.14}$ & 1 & 18.3 & 15.26 & 13.18 & 12.38 \\
\hline 249 & 9390653 & 1 & $0.40_{-0.05}^{+0.05}$ & $0.39_{-0.04}^{+0.04}$ & $3562_{-64}^{+50}$ & $-0.13_{-0.13}^{+0.13}$ & 1 & 43.6 & 14.49 & 12.00 & 11.15 \\
\hline 250 & 9757613 & 4 & $0.55_{-0.05}^{+0.05}$ & $0.52_{-0.05}^{+0.05}$ & $3884_{-27}^{+199}$ & $-0.13_{-0.13}^{+0.13}$ & 1 & 17.8 & 15.47 & 13.41 & 12.63 \\
\hline 251 & 10489206 & 2 & $0.53_{-0.03}^{+0.03}$ & $0.50_{-0.03}^{+0.03}$ & $3811_{-71}^{+48}$ & $-0.06_{-0.11}^{+0.11}$ & 1 & 14.5 & 14.75 & 12.48 & 11.68 \\
\hline 252 & 11187837 & 1 & $0.52_{-0.03}^{+0.03}$ & $0.49_{-0.03}^{+0.03}$ & $3745_{-56}^{+70}$ & $0.06_{-0.11}^{+0.11}$ & 1 & 39.5 & 15.61 & 13.42 & 12.55 \\
\hline 253 & 11752906 & 2 & $0.59_{-0.04}^{+0.04}$ & $0.56_{-0.04}^{+0.04}$ & $3759_{-34}^{+182}$ & $0.49_{-0.14}^{+0.14}$ & 1 & $\ldots$ & 15.25 & 13.09 & 12.29 \\
\hline 254 & 5794240 & 1 & $0.58_{-0.03}^{+0.03}$ & $0.55_{-0.03}^{+0.03}$ & $3793_{-34}^{+133}$ & $0.32_{-0.13}^{+0.13}$ & 1 & 15.8 & 15.98 & 13.75 & 12.89 \\
\hline 255 & 7021681 & 2 & $0.53_{-0.04}^{+0.04}$ & $0.51_{-0.04}^{+0.04}$ & $3780_{-73}^{+68}$ & $-0.01_{-0.15}^{+0.15}$ & 1 & $\cdots$ & 15.11 & 12.91 & 12.08 \\
\hline 314 & 7603200 & 3 & $0.52_{-0.03}^{+0.03}$ & $0.49_{-0.03}^{+0.03}$ & $3847_{-59}^{+46}$ & $-0.25_{-0.12}^{+0.12}$ & 1 & 19.4 & 12.93 & 10.29 & 9.51 \\
\hline 463 & 8845205 & 1 & $0.26_{-0.05}^{+0.05}$ & $0.26_{-0.04}^{+0.04}$ & $3389_{-48}^{+57}$ & $-0.12_{-0.13}^{+0.13}$ & 1 & 50.8 & 14.71 & 12.27 & 11.45 \\
\hline 478 & 10990886 & 1 & $0.54_{-0.03}^{+0.03}$ & $0.51_{-0.03}^{+0.03}$ & $3744_{-92}^{+23}$ & $0.19_{-0.12}^{+0.12}$ & 1 & 34.2 & 14.27 & 11.80 & 10.96 \\
\hline 531 & 10395543 & 1 & $0.61_{-0.04}^{+0.04}$ & $0.59_{-0.04}^{+0.04}$ & $4004_{-172}^{+120}$ & $0.11_{-0.14}^{+0.14}$ & 1 & 46.8 & 14.42 & 12.36 & 11.61 \\
\hline 571 & 8120608 & 5 & $0.48_{-0.03}^{+0.03}$ & $0.45_{-0.03}^{+0.03}$ & $3748_{-20}^{+90}$ & $-0.34_{-0.12}^{+0.12}$ & 1 & 34.3 & 14.62 & 12.47 & 11.60 \\
\hline 596 & 10388286 & 1 & $0.49_{-0.03}^{+0.03}$ & $0.46_{-0.03}^{+0.03}$ & $3670_{-53}^{+53}$ & $0.01_{-0.11}^{+0.11}$ & 1 & 37.5 & 14.82 & 12.44 & 11.57 \\
\hline 641 & 5131180 & 1 & $0.27_{-0.05}^{+0.05}$ & $0.28_{-0.05}^{+0.05}$ & $3391_{-50}^{+50}$ & $-0.10_{-0.10}^{+0.10}$ & 2 & $\ldots$ & 13.58 & 11.52 & 10.70 \\
\hline 739 & 10386984 & 1 & $0.52_{-0.04}^{+0.04}$ & $0.50_{-0.04}^{+0.04}$ & $3733_{-47}^{+91}$ & $0.11_{-0.15}^{+0.15}$ & 1 & 39.6 & 15.49 & 13.44 & 12.63 \\
\hline 781 & 11923270 & 1 & $0.50_{-0.04}^{+0.04}$ & $0.47_{-0.04}^{+0.04}$ & $3691_{-89}^{+65}$ & $-0.00_{-0.14}^{+0.14}$ & 1 & 36.4 & 15.94 & 13.47 & 12.63 \\
\hline 812 & 4139816 & 4 & $0.53_{-0.05}^{+0.05}$ & $0.51_{-0.05}^{+0.05}$ & $3949_{-130}^{+131}$ & $-0.45_{-0.14}^{+0.14}$ & 1 & 15.0 & 15.95 & 13.95 & 13.11 \\
\hline 817 & 4725681 & 2 & $0.53_{-0.03}^{+0.03}$ & $0.51_{-0.03}^{+0.03}$ & $3747_{-40}^{+81}$ & $0.11_{-0.12}^{+0.12}$ & 1 & 15.4 & 15.41 & 13.22 & 12.31 \\
\hline 818 & 4913852 & 1 & $0.54_{-0.04}^{+0.04}$ & $0.51_{-0.04}^{+0.04}$ & $3698_{-112}^{+34}$ & $0.28_{-0.16}^{+0.16}$ & 1 & 34.3 & 15.88 & 13.40 & 12.49 \\
\hline 854 & 6435936 & 1 & $0.49_{-0.04}^{+0.04}$ & $0.46_{-0.04}^{+0.04}$ & $3593_{-65}^{+37}$ & $0.25_{-0.15}^{+0.15}$ & 1 & 20.2 & 15.85 & 13.44 & 12.53 \\
\hline 886 & 7455287 & 3 & $0.48_{-0.04}^{+0.04}$ & $0.46_{-0.04}^{+0.04}$ & $3712_{-69}^{+57}$ & $-0.13_{-0.14}^{+0.14}$ & 1 & 34.6 & 15.85 & 13.51 & 12.65 \\
\hline 898 & 7870390 & 3 & $0.53_{-0.04}^{+0.04}$ & $0.51_{-0.04}^{+0.04}$ & $3907_{-104}^{+80}$ & $-0.33_{-0.13}^{+0.13}$ & 1 & 22.1 & 15.78 & 13.74 & 12.95 \\
\hline
\end{tabular}


Table 5

(Continued)

\begin{tabular}{|c|c|c|c|c|c|c|c|c|c|c|c|}
\hline KOI & KIC & $N_{\mathrm{pl}}$ & $\operatorname{Mass}\left(M_{\odot}\right)$ & Radius $\left(R_{\odot}\right)$ & $T_{\text {eff }}(\mathrm{K})$ & {$[\mathrm{Fe} / \mathrm{H}](\mathrm{dex})$} & Ref. & $P_{\text {rot }}$ (days) & $K_{p}$ & $J$ & $K_{s}$ \\
\hline 899 & 7907423 & 3 & $0.43_{-0.04}^{+0.04}$ & $0.41_{-0.04}^{+0.04}$ & $3565_{-48}^{+47}$ & $0.01_{-0.11}^{+0.11}$ & 1 & 36.1 & 15.23 & 12.84 & 11.97 \\
\hline 936 & 9388479 & 2 & $0.47_{-0.04}^{+0.04}$ & $0.45_{-0.04}^{+0.04}$ & $3582_{-44}^{+61}$ & $0.19_{-0.13}^{+0.13}$ & 1 & 36.0 & 15.07 & 12.60 & 11.72 \\
\hline 947 & 9710326 & 1 & $0.49_{-0.04}^{+0.04}$ & $0.47_{-0.04}^{+0.04}$ & $3753_{-98}^{+54}$ & $-0.26_{-0.13}^{+0.13}$ & 1 & 25.2 & 15.19 & 12.91 & 12.10 \\
\hline 952 & 9787239 & 5 & $0.51_{-0.04}^{+0.04}$ & $0.48_{-0.04}^{+0.04}$ & $3731_{-73}^{+71}$ & $-0.02_{-0.13}^{+0.13}$ & 1 & 37.1 & 15.80 & 13.61 & 12.76 \\
\hline 961 & 8561063 & 3 & $0.13_{-0.05}^{+0.05}$ & $0.17_{-0.04}^{+0.04}$ & $3204_{-40}^{+61}$ & $-0.48_{-0.12}^{+0.12}$ & 1 & $\ldots$ & 15.92 & 12.18 & 11.47 \\
\hline 1078 & 10166274 & 3 & $0.49_{-0.05}^{+0.05}$ & $0.46_{-0.04}^{+0.04}$ & $3783_{-82}^{+109}$ & $-0.34_{-0.17}^{+0.17}$ & 1 & 22.4 & 15.44 & 13.33 & 12.48 \\
\hline 1085 & 10118816 & 1 & $0.55_{-0.05}^{+0.05}$ & $0.53_{-0.05}^{+0.05}$ & $3979_{-78}^{+257}$ & $-0.33_{-0.13}^{+0.13}$ & 1 & 39.7 & 15.23 & 13.03 & 12.25 \\
\hline 1141 & 8346392 & 1 & $0.58_{-0.07}^{+0.07}$ & $0.55_{-0.06}^{+0.06}$ & $3968_{-69}^{+425}$ & $-0.14_{-0.12}^{+0.12}$ & 1 & 17.9 & 15.95 & 13.88 & 13.05 \\
\hline 1146 & 8351704 & 1 & $0.39_{-0.05}^{+0.05}$ & $0.37_{-0.04}^{+0.04}$ & $3560_{-39}^{+62}$ & $-0.18_{-0.13}^{+0.13}$ & 1 & 25.6 & 15.65 & 13.44 & 12.61 \\
\hline 1201 & 4061149 & 1 & $0.47_{-0.03}^{+0.03}$ & $0.45_{-0.03}^{+0.03}$ & $3697_{-45}^{+82}$ & $-0.19_{-0.14}^{+0.14}$ & 1 & 28.8 & 15.60 & 13.41 & 12.61 \\
\hline 1393 & 9202151 & 1 & $0.57_{-0.05}^{+0.06}$ & $0.56_{-0.05}^{+0.08}$ & $3872_{-50}^{+101}$ & $0.00_{-0.10}^{+0.10}$ & 2 & $\ldots$ & 15.80 & 13.62 & 12.77 \\
\hline 1397 & 9427402 & 1 & $0.52_{-0.06}^{+0.06}$ & $0.49_{-0.06}^{+0.06}$ & $3822_{-51}^{+169}$ & $-0.24_{-0.15}^{+0.15}$ & 1 & $\ldots$ & 15.37 & 13.25 & 12.43 \\
\hline 1408 & 9150827 & 2 & $0.57_{-0.03}^{+0.03}$ & $0.54_{-0.03}^{+0.03}$ & $3955_{-82}^{+95}$ & $-0.16_{-0.12}^{+0.12}$ & 1 & 25.0 & 14.69 & 12.66 & 11.81 \\
\hline 1422 & 11497958 & 5 & $0.39_{-0.05}^{+0.05}$ & $0.37_{-0.04}^{+0.04}$ & $3522_{-49}^{+76}$ & $-0.08_{-0.12}^{+0.12}$ & 1 & 35.6 & 15.92 & 13.39 & 12.60 \\
\hline 1427 & 11129738 & 1 & $0.54_{-0.04}^{+0.04}$ & $0.51_{-0.04}^{+0.04}$ & $3880_{-75}^{+141}$ & $-0.24_{-0.14}^{+0.14}$ & 1 & 33.8 & 15.84 & 13.80 & 13.06 \\
\hline 1649 & 11337141 & 1 & $0.58_{-0.06}^{+0.06}$ & $0.55_{-0.05}^{+0.05}$ & $3877_{-89}^{+156}$ & $0.09_{-0.13}^{+0.13}$ & 1 & 25.6 & 14.96 & 12.64 & 11.79 \\
\hline 1681 & 5531953 & 3 & $0.46_{-0.04}^{+0.04}$ & $0.43_{-0.04}^{+0.04}$ & $3657_{-64}^{+70}$ & $-0.12_{-0.14}^{+0.14}$ & 1 & 24.5 & 15.85 & 13.46 & 12.58 \\
\hline 1702 & 7304449 & 1 & $0.26_{-0.05}^{+0.05}$ & $0.27_{-0.04}^{+0.04}$ & $3356_{-57}^{+74}$ & $-0.06_{-0.14}^{+0.14}$ & 1 & 45.9 & 15.72 & 12.99 & 12.20 \\
\hline 1725 & 10905746 & 1 & $0.44_{-0.04}^{+0.04}$ & $0.42_{-0.04}^{+0.04}$ & $3618_{-41}^{+57}$ & $-0.06_{-0.12}^{+0.12}$ & 1 & 18.2 & 13.50 & 10.65 & 9.80 \\
\hline 1843 & 5080636 & 2 & $0.54_{-0.04}^{+0.04}$ & $0.51_{-0.04}^{+0.04}$ & $3705_{-81}^{+37}$ & $0.27_{-0.12}^{+0.12}$ & 1 & 34.3 & 14.40 & 11.95 & 11.06 \\
\hline 1867 & 8167996 & 3 & $0.51_{-0.04}^{+0.04}$ & $0.48_{-0.04}^{+0.04}$ & $3717_{-36}^{+123}$ & $0.03_{-0.16}^{+0.16}$ & 1 & 24.8 & 15.02 & 12.79 & 11.95 \\
\hline 1868 & 6773862 & 1 & $0.55_{-0.06}^{+0.06}$ & $0.52_{-0.06}^{+0.06}$ & $3828_{-82}^{+134}$ & $-0.05_{-0.13}^{+0.13}$ & 1 & 25.0 & 15.22 & 13.14 & 12.29 \\
\hline 1879 & 8367644 & 1 & $0.59_{-0.09}^{+0.09}$ & $0.56_{-0.08}^{+0.08}$ & $3829_{-73}^{+324}$ & $0.31_{-0.16}^{+0.16}$ & 1 & 21.9 & 15.97 & 13.59 & 12.73 \\
\hline 1880 & 10332883 & 1 & $0.57_{-0.05}^{+0.05}$ & $0.54_{-0.05}^{+0.05}$ & $3950_{-178}^{+60}$ & $-0.15_{-0.14}^{+0.14}$ & 1 & 18.9 & 14.44 & 12.26 & 11.45 \\
\hline 1902 & 5809954 & 1 & $0.46_{-0.05}^{+0.05}$ & $0.44_{-0.04}^{+0.04}$ & $3647_{-86}^{+69}$ & $-0.08_{-0.14}^{+0.14}$ & 1 & $\ldots$ & 14.65 & 12.29 & 11.45 \\
\hline 1907 & 7094486 & 1 & $0.51_{-0.05}^{+0.05}$ & $0.48_{-0.05}^{+0.05}$ & $3594_{-25}^{+113}$ & $0.39_{-0.18}^{+0.18}$ & 1 & 18.2 & 15.28 & 13.19 & 12.35 \\
\hline 2006 & 10525027 & 1 & $0.52_{-0.03}^{+0.03}$ & $0.49_{-0.03}^{+0.03}$ & $3756_{-59}^{+80}$ & $0.01_{-0.14}^{+0.14}$ & 1 & 28.8 & 14.22 & 11.97 & 11.18 \\
\hline 2036 & 6382217 & 2 & $0.55_{-0.06}^{+0.06}$ & $0.52_{-0.05}^{+0.05}$ & $3758_{-2}^{+281}$ & $0.19_{-0.13}^{+0.13}$ & 1 & 32.1 & 15.77 & 13.60 & 12.81 \\
\hline 2057 & 9573685 & 1 & $0.59_{-0.06}^{+0.06}$ & $0.56_{-0.06}^{+0.06}$ & $3974_{-188}^{+182}$ & $-0.05_{-0.16}^{+0.16}$ & 1 & 30.2 & 15.03 & 12.88 & 12.07 \\
\hline 2058 & 10329835 & 1 & $0.52_{-0.04}^{+0.04}$ & $0.49_{-0.04}^{+0.04}$ & $3747_{-50}^{+144}$ & $-0.00_{-0.15}^{+0.15}$ & 1 & 22.2 & 15.31 & 13.22 & 12.41 \\
\hline 2090 & 11348997 & 1 & $0.60_{-0.09}^{+0.09}$ & $0.57_{-0.08}^{+0.08}$ & $3889_{-141}^{+522}$ & $0.32_{-0.15}^{+0.15}$ & 1 & 36.5 & 15.53 & 13.26 & 12.39 \\
\hline 2130 & 2161536 & 1 & $0.59_{-0.06}^{+0.06}$ & $0.56_{-0.05}^{+0.05}$ & $3967_{-137}^{+206}$ & $0.06_{-0.15}^{+0.15}$ & 1 & 26.8 & 15.66 & 13.59 & 12.77 \\
\hline 2156 & 2556650 & 1 & $0.49_{-0.05}^{+0.05}$ & $0.47_{-0.04}^{+0.04}$ & $3754_{-42}^{+108}$ & $-0.26_{-0.16}^{+0.16}$ & 1 & 28.1 & 15.96 & 13.67 & 12.83 \\
\hline 2179 & 10670119 & 2 & $0.56_{-0.04}^{+0.04}$ & $0.53_{-0.04}^{+0.04}$ & $3834_{-67}^{+138}$ & $0.05_{-0.13}^{+0.13}$ & 1 & 19.6 & 15.67 & 13.25 & 12.39 \\
\hline 2191 & 5601258 & 1 & $0.57_{-0.04}^{+0.04}$ & $0.54_{-0.04}^{+0.04}$ & $3870_{-102}^{+77}$ & $0.10_{-0.13}^{+0.13}$ & 1 & 17.8 & 14.91 & 12.66 & 11.81 \\
\hline 2238 & 8229458 & 1 & $0.54_{-0.04}^{+0.04}$ & $0.51_{-0.04}^{+0.04}$ & $3809_{-90}^{+106}$ & $-0.02_{-0.14}^{+0.14}$ & 1 & 14.9 & 14.63 & 12.49 & 11.68 \\
\hline 2306 & 6666233 & 1 & $0.57_{-0.07}^{+0.07}$ & $0.54_{-0.06}^{+0.06}$ & $3861_{-64}^{+292}$ & $0.08_{-0.13}^{+0.13}$ & 1 & 24.9 & 14.78 & 12.63 & 11.83 \\
\hline 2329 & 11192235 & 1 & $0.51_{-0.05}^{+0.05}$ & $0.49_{-0.05}^{+0.05}$ & $3823_{-73}^{+138}$ & $-0.28_{-0.17}^{+0.17}$ & 1 & 34.1 & 15.65 & 13.49 & 12.67 \\
\hline 2347 & 8235924 & 1 & $0.57_{-0.08}^{+0.08}$ & $0.54_{-0.07}^{+0.07}$ & $3817_{-46}^{+510}$ & $0.24_{-0.13}^{+0.13}$ & 1 & $\ldots$ & 14.93 & 12.86 & 12.06 \\
\hline 2417 & 9654468 & 1 & $0.27_{-0.05}^{+0.05}$ & $0.28_{-0.05}^{+0.05}$ & $3391_{-50}^{+50}$ & $-0.10_{-0.10}^{+0.10}$ & 2 & 21.3 & 16.22 & 14.12 & 13.43 \\
\hline 2418 & 10027247 & 1 & $0.43_{-0.07}^{+0.05}$ & $0.41_{-0.08}^{+0.05}$ & $3724_{-74}^{+60}$ & $-0.40_{-0.10}^{+0.10}$ & 2 & 17.6 & 15.47 & 13.23 & 12.37 \\
\hline 2453 & 8631751 & 1 & $0.27_{-0.06}^{+0.06}$ & $0.27_{-0.05}^{+0.05}$ & $3451_{-51}^{+96}$ & $-0.44_{-0.16}^{+0.16}$ & 1 & $\ldots$ & 15.63 & 13.19 & 12.42 \\
\hline 2480 & 8189801 & 1 & $0.55_{-0.05}^{+0.07}$ & $0.55_{-0.05}^{+0.09}$ & $3990_{-66}^{+84}$ & $-0.20_{-0.30}^{+0.30}$ & 2 & 32.0 & 15.74 & 13.73 & 12.88 \\
\hline 2542 & 6183511 & 1 & $0.37_{-0.06}^{+0.06}$ & $0.36_{-0.06}^{+0.06}$ & $3466_{-70}^{+66}$ & $0.10_{-0.15}^{+0.15}$ & 1 & 29.4 & 15.53 & 12.86 & 12.03 \\
\hline 2626 & 11768142 & 1 & $0.43_{-0.06}^{+0.06}$ & $0.40_{-0.05}^{+0.05}$ & $3568_{-105}^{+52}$ & $-0.02_{-0.16}^{+0.16}$ & 1 & $\ldots$ & 15.93 & 13.45 & 12.63 \\
\hline 2650 & 8890150 & 2 & $0.52_{-0.04}^{+0.04}$ & $0.50_{-0.04}^{+0.04}$ & $3765_{-49}^{+96}$ & $0.01_{-0.13}^{+0.13}$ & 1 & 19.9 & 15.99 & 13.82 & 12.95 \\
\hline 2662 & 3426367 & 1 & $0.48_{-0.05}^{+0.05}$ & $0.46_{-0.05}^{+0.05}$ & $3628_{-74}^{+57}$ & $0.14_{-0.14}^{+0.14}$ & 1 & 34.0 & 14.49 & 11.88 & 11.02 \\
\hline 2704 & 9730163 & 2 & $0.24_{-0.06}^{+0.06}$ & $0.26_{-0.05}^{+0.05}$ & $3267_{-54}^{+70}$ & $0.27_{-0.15}^{+0.15}$ & 1 & $\ldots$ & 17.48 & 13.54 & 12.61 \\
\hline 2705 & 11453592 & 1 & $0.39_{-0.05}^{+0.05}$ & $0.38_{-0.05}^{+0.05}$ & $3494_{-54}^{+55}$ & $0.11_{-0.14}^{+0.14}$ & 1 & $\ldots$ & 14.72 & 11.57 & 10.73 \\
\hline 2715 & 9837661 & 3 & $0.50_{-0.08}^{+0.08}$ & $0.47_{-0.07}^{+0.07}$ & $3640_{-74}^{+291}$ & $0.20_{-0.20}^{+0.20}$ & 1 & 41.3 & 16.83 & 14.83 & 13.88 \\
\hline 2764 & 10073672 & 1 & $0.59_{-0.11}^{+0.11}$ & $0.57_{-0.11}^{+0.11}$ & $4045_{-114}^{+* * * *}$ & $-0.16_{-0.16}^{+0.16}$ & 1 & 27.7 & 15.79 & 13.67 & 12.87 \\
\hline 2793 & 9823519 & 2 & $0.47_{-0.05}^{+0.08}$ & $0.44_{-0.05}^{+0.07}$ & $3698_{-50}^{+75}$ & $-0.20_{-0.20}^{+0.20}$ & 2 & 19.0 & 16.28 & 13.99 & 13.12 \\
\hline 2839 & 6186964 & 1 & $0.58_{-0.08}^{+0.08}$ & $0.55_{-0.08}^{+0.08}$ & $3943_{-184}^{+178}$ & $-0.05_{-0.17}^{+0.17}$ & 1 & 17.8 & 15.88 & 13.77 & 12.96 \\
\hline 2842 & 8733898 & 3 & $0.16_{-0.02}^{+0.02}$ & $0.18_{-0.02}^{+0.02}$ & $3204_{-57}^{+51}$ & $-0.30_{-0.13}^{+0.13}$ & 1 & $\ldots$ & 16.26 & 13.59 & 12.83 \\
\hline 2845 & 10591855 & 1 & $0.72_{-0.11}^{+0.11}$ & $0.67_{-0.11}^{+0.11}$ & $4342_{-530}^{+712}$ & $0.32_{-0.17}^{+0.17}$ & 1 & 29.1 & 15.57 & 13.51 & 12.70 \\
\hline 2862 & 6679295 & 1 & $0.52_{-0.06}^{+0.06}$ & $0.50_{-0.06}^{+0.06}$ & $3679_{-44}^{+146}$ & $0.26_{-0.18}^{+0.18}$ & 1 & 17.7 & 15.91 & 13.66 & 12.76 \\
\hline 2926 & 10122538 & 4 & $0.60_{-0.09}^{+0.09}$ & $0.57_{-0.09}^{+0.09}$ & $3899_{-110}^{+717}$ & $0.24_{-0.17}^{+0.17}$ & 1 & 24.0 & 16.28 & 14.22 & 13.34 \\
\hline
\end{tabular}


Table 5

(Continued)

\begin{tabular}{|c|c|c|c|c|c|c|c|c|c|c|c|}
\hline KOI & KIC & $N_{\mathrm{pl}}$ & Mass $\left(M_{\odot}\right)$ & Radius $\left(R_{\odot}\right)$ & $T_{\text {eff }}(\mathrm{K})$ & {$[\mathrm{Fe} / \mathrm{H}](\mathrm{dex})$} & Ref. & $P_{\text {rot }}($ days $)$ & $K_{p}$ & $J$ & $K_{s}$ \\
\hline 2992 & 8509442 & 1 & $0.57_{-0.05}^{+0.09}$ & $0.55_{-0.05}^{+0.08}$ & $3952_{-55}^{+93}$ & $-0.10_{-0.30}^{+0.30}$ & 2 & 15.0 & 15.99 & 13.91 & 13.04 \\
\hline 3010 & 3642335 & 1 & $0.53_{-0.09}^{+0.09}$ & $0.51_{-0.08}^{+0.08}$ & $3810_{-270}^{+54}$ & $-0.03_{-0.24}^{+0.24}$ & 1 & 14.5 & 15.76 & 13.59 & 12.77 \\
\hline 3034 & 2973386 & 1 & $0.49_{-0.06}^{+0.06}$ & $0.46_{-0.06}^{+0.06}$ & $3713_{-44}^{+230}$ & $-0.10_{-0.18}^{+0.18}$ & 1 & 37.2 & 15.57 & 13.45 & 12.61 \\
\hline 3094 & 12352520 & 1 & $0.53_{-0.05}^{+0.05}$ & $0.50_{-0.05}^{+0.05}$ & $3805_{-50}^{+68}$ & $-0.10_{-0.10}^{+0.10}$ & 2 & 36.2 & 15.74 & 13.52 & 12.75 \\
\hline 3102 & 9605552 & 1 & $0.38_{-0.11}^{+0.07}$ & $0.35_{-0.12}^{+0.06}$ & $3773_{-134}^{+50}$ & $-0.80_{-0.20}^{+0.20}$ & 2 & 31.0 & 15.98 & 13.86 & 13.10 \\
\hline 3119 & 5551672 & 1 & $0.22_{-0.07}^{+0.07}$ & $0.23_{-0.06}^{+0.06}$ & $3371_{-125}^{+75}$ & $-0.40_{-0.21}^{+0.21}$ & 1 & $\ldots$ & 16.95 & 14.38 & 13.54 \\
\hline 3140 & 5978170 & 1 & $0.27_{-0.05}^{+0.05}$ & $0.28_{-0.05}^{+0.05}$ & $3391_{-50}^{+50}$ & $-0.10_{-0.10}^{+0.10}$ & 2 & 15.2 & 15.56 & 13.52 & 12.77 \\
\hline 3144 & 5688790 & 1 & $0.45_{-0.05}^{+0.05}$ & $0.42_{-0.05}^{+0.05}$ & $3679_{-99}^{+67}$ & $-0.31_{-0.18}^{+0.18}$ & 1 & 31.7 & 16.11 & 13.58 & 12.74 \\
\hline 3263 & 11853130 & 1 & $0.49_{-0.06}^{+0.06}$ & $0.46_{-0.05}^{+0.05}$ & $3680_{-78}^{+128}$ & $0.00_{-0.17}^{+0.17}$ & 1 & 19.9 & 15.95 & 13.56 & 12.73 \\
\hline 3282 & 12066569 & 1 & $0.55_{-0.07}^{+0.07}$ & $0.53_{-0.07}^{+0.07}$ & $3901_{-106}^{+264}$ & $-0.20_{-0.14}^{+0.14}$ & 1 & 18.0 & 15.85 & 13.77 & 12.92 \\
\hline 3284 & 6497146 & 1 & $0.55_{-0.04}^{+0.04}$ & $0.52_{-0.04}^{+0.04}$ & $3748_{-112}^{+35}$ & $0.16_{-0.14}^{+0.14}$ & 1 & 36.5 & 14.47 & 12.13 & 11.20 \\
\hline 3414 & 6023859 & 1 & $0.56_{-0.05}^{+0.09}$ & $0.54_{-0.05}^{+0.09}$ & $3900_{-50}^{+140}$ & $-0.10_{-0.20}^{+0.20}$ & 2 & 14.5 & 15.48 & 13.37 & 12.52 \\
\hline 3444 & 5384713 & 4 & $0.51_{-0.04}^{+0.04}$ & $0.48_{-0.04}^{+0.04}$ & $3665_{-82}^{+88}$ & $0.16_{-0.14}^{+0.14}$ & 1 & 20.3 & 13.69 & 11.17 & 10.31 \\
\hline 3497 & 8424002 & 1 & $0.32_{-0.06}^{+0.06}$ & $0.32_{-0.05}^{+0.05}$ & $3412_{-106}^{+50}$ & $0.02_{-0.17}^{+0.17}$ & 1 & 9.4 & 13.39 & 11.31 & 10.62 \\
\hline 3749 & 11547869 & 1 & $0.28_{-0.07}^{+0.07}$ & $0.29_{-0.06}^{+0.06}$ & $3337_{-55}^{+130}$ & $0.10_{-0.18}^{+0.18}$ & 1 & 19.5 & 16.42 & 13.73 & 12.97 \\
\hline 4087 & 6106282 & 1 & $0.48_{-0.07}^{+0.05}$ & $0.48_{-0.06}^{+0.06}$ & $3813_{-87}^{+58}$ & $-0.30_{-0.10}^{+0.10}$ & 2 & 17.5 & 15.13 & 12.96 & 12.12 \\
\hline 4252 & 10525049 & 1 & $0.58_{-0.06}^{+0.06}$ & $0.55_{-0.06}^{+0.06}$ & $3873_{-76}^{+275}$ & $0.12_{-0.12}^{+0.12}$ & 1 & 30.9 & 13.98 & 11.70 & 10.87 \\
\hline 4290 & 5868793 & 1 & $0.18_{-0.04}^{+0.04}$ & $0.20_{-0.04}^{+0.04}$ & $3187_{-84}^{+78}$ & $0.10_{-0.17}^{+0.17}$ & 1 & $\ldots$ & 17.06 & 13.28 & 12.44 \\
\hline 4427 & 4172805 & 1 & $0.53_{-0.06}^{+0.06}$ & $0.50_{-0.06}^{+0.06}$ & $3813_{-30}^{+272}$ & $-0.07_{-0.14}^{+0.14}$ & 1 & 39.0 & 15.65 & 13.34 & 12.50 \\
\hline 4875 & 2986833 & 1 & $0.58_{-0.08}^{+0.08}$ & $0.56_{-0.08}^{+0.08}$ & $3874_{-174}^{+283}$ & $0.15_{-0.17}^{+0.17}$ & 1 & 30.4 & 15.78 & 13.48 & 12.64 \\
\hline 5228 & 6036286 & 1 & $0.37_{-0.05}^{+0.07}$ & $0.36_{-0.05}^{+0.06}$ & $3434_{-73}^{+72}$ & $0.00_{-0.10}^{+0.10}$ & 2 & 53.6 & 15.35 & 12.77 & 11.94 \\
\hline 5359 & 7130612 & 1 & $0.55_{-0.05}^{+0.05}$ & $0.53_{-0.05}^{+0.05}$ & $3939_{-51}^{+52}$ & $-0.20_{-0.10}^{+0.10}$ & 2 & 16.3 & 16.56 & 14.41 & 13.59 \\
\hline 5692 & 9575728 & 1 & $0.55_{-0.06}^{+0.05}$ & $0.54_{-0.05}^{+0.05}$ & $3901_{-95}^{+50}$ & $-0.10_{-0.10}^{+0.10}$ & 2 & 39.8 & 14.14 & 12.00 & 11.18 \\
\hline
\end{tabular}

\section{Cumulative Stellar Parameter Distributions}
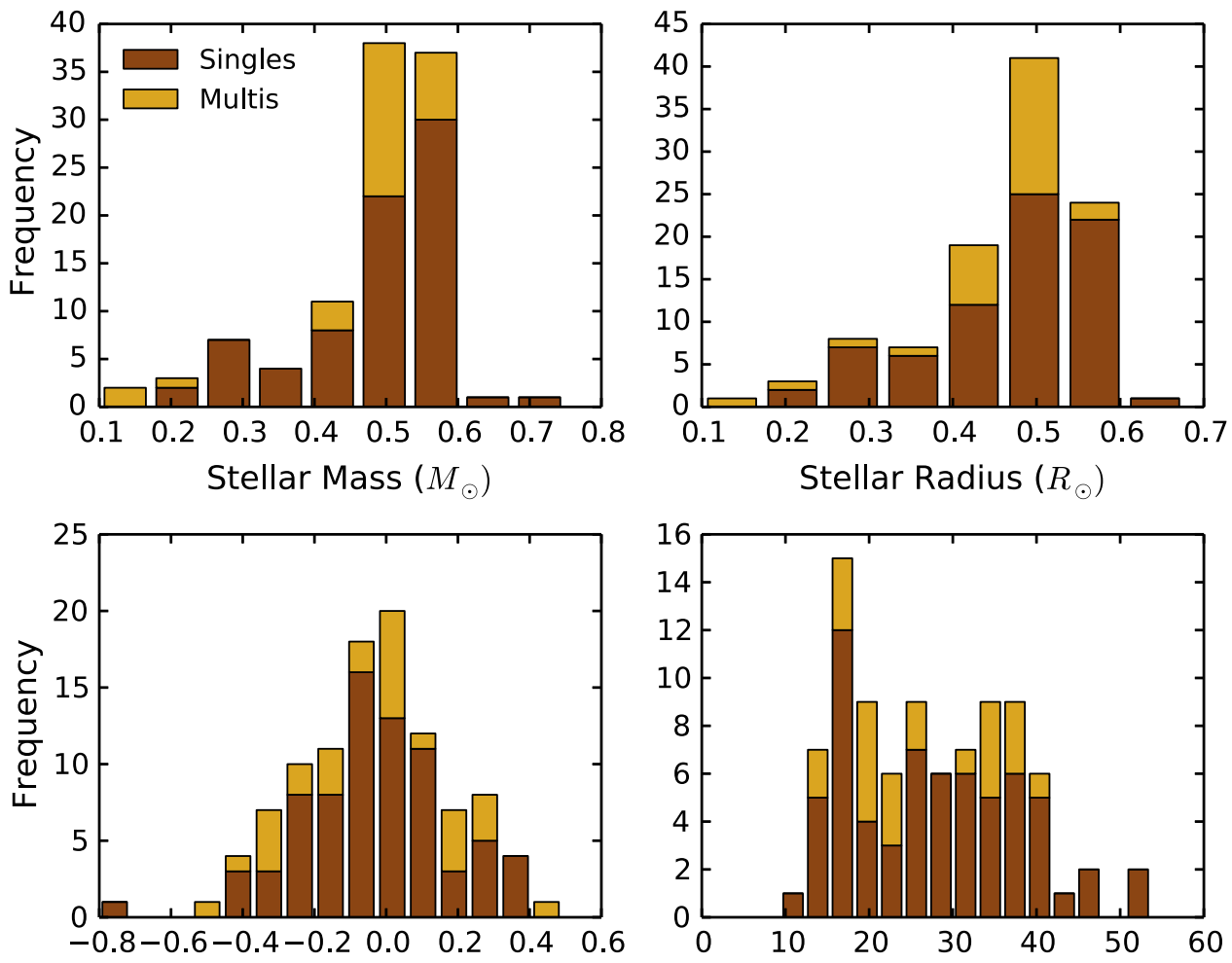

$[\mathrm{Fe} / \mathrm{H}](\mathrm{dex})$

Stellar Rotation Period (d)

Figure 10. Distributions of stellar parameters for the final ensemble of 104 cool KOIs. The host stars of single transit systems and multi-transit systems have been distinguished and are shown in dark and light shading, respectively. 
The cumulative distributions for the four transit parameters that are most relevant to the planet statistics are displayed in Figure 9. For this plot and those that follow, we distinguish between the planets that are in single transit and multi-transit systems.

\subsection{Stellar Characteristics}

The physical parameters of the transiting planets are intimately tied to the stellar parameters. We therefore also consolidate data for the stellar sample both from this work and from the literature. Stellar masses, radii, and effective temperatures were obtained from the lists of Muirhead et al. (2014) and Dressing \& Charbonneau (2013). By default, we use the stellar parameters derived from the medium-resolution, infrared spectroscopy of Muirhead et al. (2014). The method uses a calibrated empirical relationship between the shape of the pseudo-continuum in the $K$-band spectrum to infer a stellar effective temperature $\left(\mathrm{H}_{2} \mathrm{O}-\mathrm{K} 2\right.$ index; Rojas-Ayala et al. 2012). The equivalent widths of the $\mathrm{Ca}$ I triplet and $\mathrm{Na}$ doublet within the same band are used to estimate the stellar metallicity using a relationship calibrated on nearby wide binaries with FGK type stars (Rojas-Ayala et al. 2010). The mass and radius of the star are then estimated by interpolating these $T_{\text {eff }}$ and $[\mathrm{M} / \mathrm{H}]$ values onto stellar evolutionary tracks (Dotter et al. 2008; Feiden et al. 2011).

For KOIs that do not have parameters derived with nearinfrared spectra, we use the stellar parameters from Dressing \& Charbonneau (2013). Here, the authors interpolate the wide band photometry from the KIC (Brown et al. 2011) onto stellar evolution models to obtain masses, radii, and metallicities. The mass and radius values derived by this method are typically in reasonable agreement with Muirhead et al. (2014), while the metallicity estimates are comparatively less reliable.

These compiled values and errors are presented in Table 5 along with the photometry from the KIC. In addition to this information, we also include our estimate of the stellar rotation period derived from the rotational modulation of an inhomogeneous surface brightness distribution. We are able to detect this rotational signature in a large fraction of our sample, about $86 \%$, and report the period corresponding to the largest peak of the auto-correlation function that we validate by visual inspection. The stellar rotation period can be an important parameter in the characterization of the planet sample as this allows for age estimates (Barnes 2003) as well as activity levels (e.g., Reiners et al. 2012). The distribution of stellar parameters is shown for host stars of single and multi-transit systems in Figure 10.

\section{SUMMARY AND CONCLUSION}

Many exciting discoveries and insights from the Kepler Mission have come from the relatively small sample of $\mathrm{M}$ dwarf stars (Johnson et al. 2011b, 2012; Muirhead et al. 2012b, 2013; Dressing \& Charbonneau 2013; Ballard \& Johnson 2014; Kipping et al. 2014; Morton \& Swift 2014; Quintana et al. 2014). The small sizes of these stars make it easier to probe deeper into the realm of super-Earth and terrestrial planets where planets form most readily. The cool surface temperatures facilitate detections of ever smaller planets in or near where liquid water may exist on their surfaces due to the shorter orbital periods and higher transit probability. While this sample is a mere $2 \%$ of the total number of stars Kepler observed during its primary mission, it offers a glimpse into the formation of the most numerous planets orbiting the most numerous stars in the Galaxy.

These facts have played a large role in motivating our group's efforts to understand this population of stars and planets. In this work, we present a uniform analysis of the photometry of cool dwarf stars spanning the full Kepler primary mission, the results of which are catalogs of transit parameters and stellar parameters for 163 transit candidates orbiting 104 low-mass dwarf stars. The stellar parameters are taken primarily from Muirhead et al. (2014) and are supplemented with values from Dressing \& Charbonneau (2013). We add new stellar rotation periods estimated directly from the Kepler light curves, and recover rotational modulation for approximately $86 \%$ of our targets.

As the statistical treatments of the Kepler data set continue to advance and improve, these transit parameters are meant to serve as a valuable data set. To facilitate further studies, we provide the posterior distributions of the transit parameters for each planet candidate including short cadence fit parameters where available. Diagnostic plots for each KOI created during the reduction and analysis of the light curves are also available for each star and transit.

J.J.S. would like to thank Jason Eastman, David Kipping, Ellen Price, and Natalie Batalha for their helpful input regarding various aspects of this work. All of the data presented in this paper were obtained from the MAST. STScI is operated by the Association of Universities for Research in Astronomy, Inc., under NASA contract NAS5-26555. Support for MAST for non-Hubble Space Telescope data is provided by the NASA Office of Space Science via grant NNX13AC07G and by other grants and contracts. This paper includes data collected by the Kepler mission. Funding for the Kepler mission is provided by the NASA Science Mission directorate. A.V. and B.T.M. are supported by the National Science Foundation Graduate Research Fellowship, grant No. DGE 1144152 and DGE 1144469, respectively.

\section{REFERENCES}

Agol, E., Steffen, J., Sari, R., \& Clarkson, W. 2005, MNRAS, 359, 567

Akeson, R. L., Chen, X., Ciardi, D., et al. 2013, PASP, 125, 989

Ballard, S., \& Johnson, J. A. 2014, arXiv:1410.4192

Barnes, S. A. 2003, ApJ, 586, 464

Batalha, N. M., Rowe, J. F., Bryson, S. T., et al. 2013, ApJS, 204, 24

Batygin, K., \& Morbidelli, A. 2013, AJ, 145, 1

Borucki, W. J., Koch, D., Basri, G., et al. 2010, Sci, 327, 977

Borucki, W. J., Koch, D. G., Basri, G., et al. 2011a, ApJ, 728, 117

Borucki, W. J., Koch, D. G., Basri, G., et al. 2011b, ApJ, 736, 19

Brown, T. M., Latham, D. W., Everett, M. E., \& Esquerdo, G. A. 2011, AJ, 142,112

Burke, C. J., Bryson, S. T., Mullally, F., et al. 2014, ApJS, 210, 19

Carter, J. A., \& Winn, J. N. 2009, ApJ, 704, 51

Chiang, E., \& Laughlin, G. 2013, MNRAS, 431, 3444

Christiansen, J. L., Clarke, B. D., Burke, C. J., et al. 2013, ApJS, 207, 35 Coughlin, J. L., Thompson, S. E., Bryson, S. T., et al. 2014, AJ, 147, 119

Dotter, A., Chaboyer, B., Jevremović, D., et al. 2008, ApJS, 178, 89

Dressing, C. D., \& Charbonneau, D. 2013, ApJ, 767, 95

Fabrycky, D. C., Ford, E. B., Steffen, J. H., et al. 2012, ApJ, 750, 114

Fabrycky, D. C., Lissauer, J. J., Ragozzine, D., et al. 2014, ApJ, 790, 146

Fang, J., \& Margot, J.-L. 2012, ApJ, 761, 92

Feiden, G. A., Chaboyer, B., \& Dotter, A. 2011, ApJL, 740, L25

Fischer, D. A., Schwamb, M. E., Schawinski, K., et al. 2012, MNRAS, 419, 2900

Ford, E. B., Rowe, J. F., Fabrycky, D. C., et al. 2011, ApJS, 197, 2

Foreman-Mackey, D., Hogg, D. W., Lang, D., \& Goodman, J. 2013, PASP, 125,306 
Foreman-Mackey, D., Hogg, D. W., \& Morton, T. D. 2014, ApJ, 795, 64 Fressin, F., Torres, G., Charbonneau, D., et al. 2013, ApJ, 766, 81

Goldreich, P., \& Schlichting, H. E. 2014, AJ, 147, 32

Hansen, B. M. S., \& Murray, N. 2012, ApJ, 751, 158

Henry, T. J., Kirkpatrick, J. D., \& Simons, D. A. 1994, AJ, 108, 1437

Henry, T. J., Subasavage, J. P., Brown, M. A., et al. 2004, AJ, 128, 2460

Holman, M. J., \& Murray, N. W. 2005, Sci, 307, 1288

Howard, A. W., Marcy, G. W., Bryson, S. T., et al. ApJS, 201, 15

Jenkins, J. M., Caldwell, D. A., Chandrasekaran, H., et al. 2010, ApJL, 713, L87

Johnson, J. A., Apps, K., Gazak, J. Z., et al. 2011, ApJ, 730, 79

Johnson, J. A., Gazak, J. Z., Apps, K., et al. 2012, AJ, 143, 111

Kipping, D. M. 2010a, MNRAS, 408, 1758

Kipping, D. M. 2010b, MNRAS, 407, 301

Kipping, D. M. 2013, MNRAS, 435, 2152

Kipping, D. M., Nesvorný, D., Buchhave, L. A., et al. 2014, ApJ, 784, 28

Koch, D. G., Borucki, W. J., Basri, G., et al. 2010, ApJL, 713, L79

Kovács, G., Zucker, S., \& Mazeh, T. 2002, A\&A, 391, 369

Lissauer, J. J., Fabrycky, D. C., Ford, E. B., et al. 2011a, Natur, 470, 53

Lissauer, J. J., Marcy, G. W., Bryson, S. T., et al. 2014, ApJ, 784, 44

Lissauer, J. J., Ragozzine, D., Fabrycky, D. C., et al. 2011b, ApJS, 197, 8

Lithwick, Y., \& Wu, Y. 2012, ApJL, 756, L11

Mandel, K., \& Agol, E. 2002, ApJL, 580, L171

Mann, A. W., Gaidos, E., \& Ansdell, M. 2013, ApJ, 779, 188

Mann, A. W., Gaidos, E., Lépine, S., \& Hilton, E. J. 2012, ApJ, 753, 90

Markwardt, C. B. 2009, in ASP Conf. Ser. 411, Astronomical Data

Analysis Software and Systems XVIII, ed. D. A. Bohlender, D. Durand, \&

P. Dowler (San Francisco, CA: ASP), 251

Mazeh, T., Nachmani, G., Holczer, T., et al. 2013, ApJS, 208, 16

Morton, T. D. 2012, ApJ, 761, 6

Morton, T. D., \& Johnson, J. A. 2011, ApJ, 738, 170

Morton, T. D., \& Swift, J. 2014, ApJ, 791, 10

Muirhead, P. S., Becker, J., Feiden, G. A., et al. 2014, ApJS, 213, 5

Muirhead, P. S., Hamren, K., Schlawin, E., et al. 2012a, ApJL, 750, L37

Muirhead, P. S., Johnson, J. A., \& Apps, K. 2012b, ApJ, 747, 144
Muirhead, P. S., Mann, A. W., Vanderburg, A., et al. 2015, arXiv

Muirhead, P. S., Vanderburg, A., Shporer, A., et al. 2013, ApJ, 767, 111

Mullally, F., Coughlin, J. L., Thompson, S. E., et al. 2015, ApJS, 217, 31

Newton, E. R., Charbonneau, D., Irwin, J., \& Mann, A. W. 2015, ApJ, 800, 85

Ofir, A. 2014, A\&A, 561, A138

Ofir, A., \& Dreizler, S. 2013, A\&A, 555, A58

Petigura, E. A., Howard, A. W., \& Marcy, G. W. 2013a, PNAS, 110, 19273

Petigura, E. A., Marcy, G. W., \& Howard, A. W. 2013b, ApJ, 770, 69

Pont, F., Zucker, S., \& Queloz, D. 2006, MNRAS, 373, 231

Price, E. M., \& Rogers, L. A. 2014, ApJ, 794, 92

Quintana, E. V., Barclay, T., Raymond, S. N., et al. 2014, Sci, 344, 277

Reid, I. N., \& Cruz, K. L. 2002, AJ, 123, 2806

Rein, H. 2012, MNRAS, 427, L21

Reiners, A., Joshi, N., \& Goldman, B. 2012, AJ, 143, 93

Rodríguez-López, C., MacDonald, J., \& Moya, A. 2012, MNRAS, 419, L44

Rojas-Ayala, B., Covey, K. R., Muirhead, P. S., \& Lloyd, J. P. 2010, ApJL, 720, L113

Rojas-Ayala, B., Covey, K. R., Muirhead, P. S., \& Lloyd, J. P. 2012, ApJ, 748, 93

Rowe, J. F., Bryson, S. T., Marcy, G. W., et al. 2014, ApJ, 784, 45

Rowe, J. F., Coughlin, J. L., Antoci, V., et al. 2015, ApJS, 217, 16

Sanchis-Ojeda, R., Rappaport, S., Winn, J. N., et al. 2014, ApJ, 787, 47

Schlichting, H. E. 2014, ApJL, 795, L15

Seager, S., \& Mallén-Ornelas, G. 2003, ApJ, 585, 1038

Smith, J. C., Stumpe, M. C., van Cleve, J. E., et al. 2012, PASP, 124, 1000

Steffen, J. H., Fabrycky, D. C., Agol, E., et al. 2013, MNRAS, 428, 1077

Stumpe, M. C., Smith, J. C., van Cleve, J. E., et al. 2012, PASP, 124, 985

Swift, J. J., Johnson, J. A., Morton, T. D., et al. 2013, ApJ, 764, 105

Szabó, R., Szabó, G. M., Dálya, G., et al. 2013, A\&A, 553, A17

Tremaine, S., \& Dong, S. 2012, AJ, 143, 94

Winn, J. N., Holman, M. J., Torres, G., et al. 2008, ApJ, 683, 1076

Wu, H., Twicken, J. D., Tenenbaum, P., et al. 2010, Proc. SPIE, 7740, 19

Wu, Y., \& Lithwick, Y. 2013, ApJ, 772, 74 